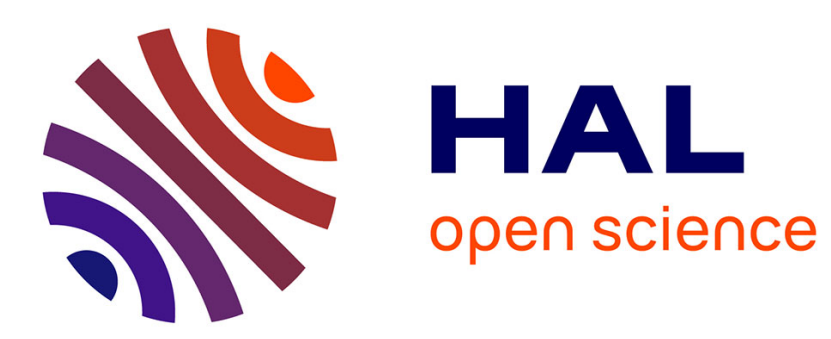

\title{
Generalized Impedance Boundary Conditions for Thin Dielectric Coatings with Variable Thickness
}

\author{
Birol Aslanyurek, Houssem Haddar, Hulya Sahinturk
}

\section{To cite this version:}

Birol Aslanyurek, Houssem Haddar, Hulya Sahinturk. Generalized Impedance Boundary Conditions for Thin Dielectric Coatings with Variable Thickness. [Research Report] RR-7145, INRIA. 2009. inria-00440159

\section{HAL Id: inria-00440159 \\ https://hal.inria.fr/inria-00440159}

Submitted on 9 Dec 2009

HAL is a multi-disciplinary open access archive for the deposit and dissemination of scientific research documents, whether they are published or not. The documents may come from teaching and research institutions in France or abroad, or from public or private research centers.
L'archive ouverte pluridisciplinaire HAL, est destinée au dépôt et à la diffusion de documents scientifiques de niveau recherche, publiés ou non, émanant des établissements d'enseignement et de recherche français ou étrangers, des laboratoires publics ou privés. 
INSTITUT NATIONAL DE RECHERCHE EN INFORMATIQUE ET EN AUTOMATIQUE

\section{Generalized Impedance Boundary Conditions for} Thin Dielectric Coatings with Variable Thickness

Birol Aslanyürek — Houssem Haddar — Hülya Şahintürk

\section{$\mathbf{N}^{\circ} \mathbf{7 1 4 5}$}

December 2009

Thème NUM

\section{apport}





\title{
Generalized Impedance Boundary Conditions for Thin Dielectric Coatings with Variable Thickness
}

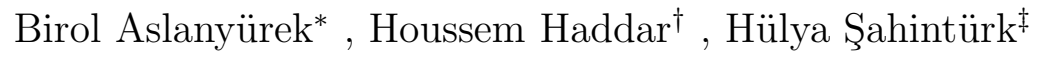 \\ Thème NUM - Systèmes numériques \\ Équipe-Projet DeFI \\ Rapport de recherche $n^{\circ} 7145$ - December 2009 - 53 pages
}

\begin{abstract}
We derive so-called Generalized Impedance Boundary Conditions (GIBC) that model thin dielectric coatings with variable width. We treat the 2-D electromagnetic problem for both TM and TE polarizations. The expressions of the GIBCs are explicited up to the third order (with respect to the coating width). The order of convergence is numerically validated through various numerical examples. A particular attention is given to the cases where the inner boundary has corner singularities.
\end{abstract}

Key-words: Scattering problems, Electromagnetism, Asymptotic models, generalized impedance boundary conditions, GIBC, thin coatings

* Mathematical Engineering Department, Yildiz Technical University, Davutpasa, Istanbul, Turkey

$\dagger$ INRIA Saclay Ile de France / CMAP Ecole Polytechnique, Route de Saclay, 91128 Palaiseau Cedex FRANCE

‡ Mathematical Engineering Department, Yildiz Technical University, Davutpasa, Istanbul, Turkey

Centre de recherche INRIA Saclay - Île-de-France

Parc Orsay Université

4, rue Jacques Monod, 91893 ORSAY Cedex

Téléphone : +33172925900 


\section{Impédances Généralisées pour des revêtements diélectriques d'épaisseurs variables}

Résumé : Nous dérivons des conditions d'impédances généralisées (GIBC) qui modélisent la présence d'un revêtement diélectrique d'épaisseur variable. Nous traitons le cas des ondes électromagnétiques 2-D aussi bien pour une polarisation TM (transverse magnétique) que TE (transverse électrique). Les expressions des GIBC sont explicitées jusqu'à l'ordre 3 (par rapport à l'épaisseur du revêtement). L'ordre de convergence est validé numériquement à travers diverses expérimentations. Une attention particulière est donnée au cas où la géométrie intérieure comporte des singularités de type coins.

Mots-clés : Problèmes de diffraction, Electromagnétisme, Modèles asymptotiques, Conditions d'Impédances Généralisées, GIBC, revêtements de faible épaisseur 


\section{Introduction}

We are interested in time harmonic electromagnetic scattering problems where the scattering object is made of a perfect conductor coated with a dielectric layer whose width $\delta$ is small compared to the incident wavelength. It is well known that a possible approach to overcome the numerical difficulties caused by the small thickness of the coating is the use of so-called Generalized Impedance Boundary Conditions (GIBC) [5, 2, 1,. These conditions lead to approximate models that are close to the original one up to an $O\left(\delta^{k+1}\right)$ error, where $k$ denotes the order of the GIBC. They are numerically attractive since associated scattering problems are formulated only on the domain exterior to the coating, and therefore do not require a meshing of the thin layer.

In order to widen applicative perspectives of GIBCs we shall address, in the present work, configurations where the coating can have a variable width (localized coatings, corrugated surfaces, ...). These cases are more technical than the case of coatings with constant width (that can be found in the classical literature on the subject) and lead to non intuitive expressions of the GIBCs. We shall restrict ourselves in these first investigations to the 2-D problem but consider both possible polarizations of the incident electromagnetic wave: TM corresponding to a Dirichlet boundary condition on the perfect scatterer and TE corresponding to a Neumann boundary condition. In order to derive these expressions we adopted a formalism similar to 3, 4] based on so-called scaled asymptotic expansions. Semi-analytical expressions of the expansion are obtained for each polarization till the third order. Let us however emphasize that while a notable difference can be observed in the derivation of the GIBC expressions, the theoretical justification of the obtained models (i.e. derivation of error estimates) would follow the same lines as in the constant case and for sufficiently regular geometries (see for instance 1]). We therefore shall only concentrate on the numerical validation of obtained models. With that perspective, number of experiments are conducted to check the formally predicted convergence rate for the derived models. A particular attention is given to the case where the interior boundary of the coating is not regular (the outer boundary is however assumed to be regular). We explain how the expressions of the GIBC can be adapted to these cases and numerically test that the adaptation preserve the formally predicted rate of convergence for this case.

The document is organized as follows. The next section is devoted to a presentation of the mathematical model associated with the "exact" scattering problem and introduces the concept of GIBC together with some useful tools of differential geometry. The third section is dedicated to the derivation of GIBC expressions using the method of scaled asymptotic expansions. The last sections contain validating numerical results and discussions of the cases where the geometry can have some singularities of corners type.

\section{Formulation of The Problem}

Consider the scatterer illustrated in Figure 1 In this configuration a homogeneous, lossy, non-magnetic, dielectric material denoted by $\Omega_{+}^{\delta}$ is coated on a perfectly electric conductor (PEC) or perfect magnetic conductor (PMC) denoted by $\Omega^{\delta}$. Background medium is symbolized by $\Omega_{-}$. $\Gamma$ symbolizes the outer 


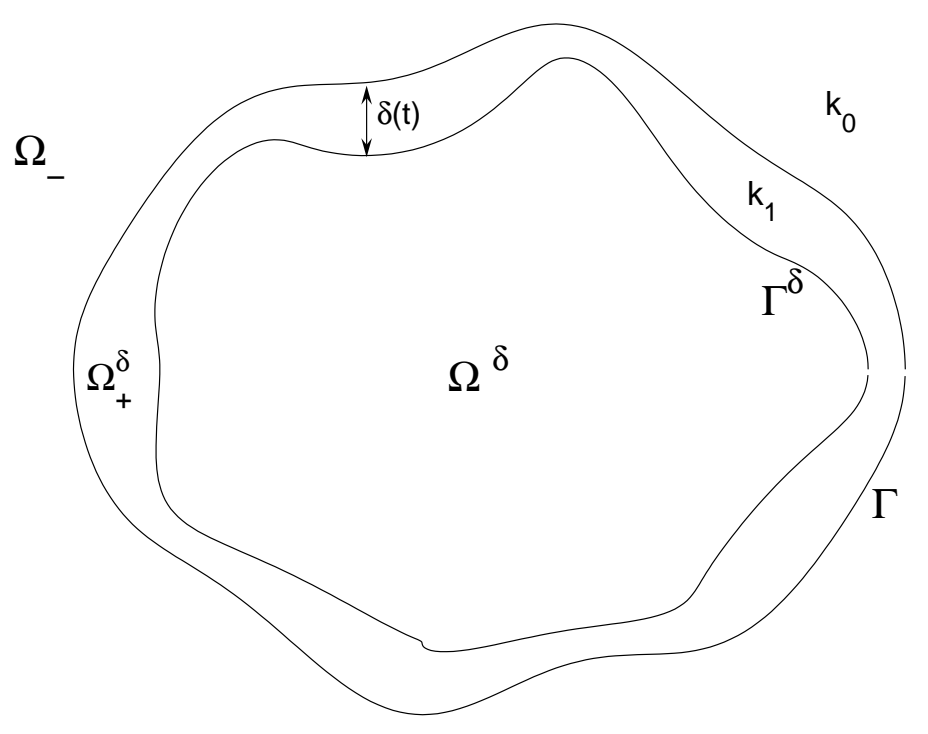

Figure 1: Geometry of thin coating with variable width

boundary of $\Omega_{+}^{\delta}$ while $\Gamma^{\delta}$ symbolizes boundary of $\Omega^{\delta}$. Now we consider the scattering problem related to the coated object. In this case the total field in the whole space satisfies reduced wave equation

$$
\Delta u_{-}^{\delta}+k^{2} u_{-}^{\delta}=0 \quad \text { in } \Omega_{-}
$$

and

$$
\Delta u_{+}^{\delta}+k^{2} u_{+}^{\delta}=0 \quad \text { in } \Omega_{+}^{\delta}
$$

where $k$ is the wave number defined by

$$
k= \begin{cases}k_{0}=\omega \sqrt{\varepsilon_{0} \mu_{0}} & \text { in } \Omega_{-} \\ k_{1}=\sqrt{\omega^{2} \varepsilon_{1} \mu_{0}+i \omega \sigma_{1} \mu_{0}} & \text { in } \Omega_{+}^{\delta}\end{cases}
$$

where $\varepsilon_{0}$ and $\varepsilon_{1}$ are the dielectric permittivity of background medium and the coating, $\sigma_{1}$ is conductivity of the coating, $\mu_{0}$ is the magnetic constant of the background medium. $u_{-}^{\delta}$ is defined as

$$
u_{-}^{\delta}=u^{i}+u_{s}^{\delta} \quad \text { in } \Omega_{-}
$$

Here $u^{i}$ is the incident wave while $u_{s}^{\delta}$ is the scattered field. $u_{s}^{\delta}$ also satisfies the Sommerfeld Radiation Condition (RC). Also,

$$
u_{-}^{\delta}=u_{+}^{\delta} \quad \text { on } \Gamma
$$

and

$$
\frac{\partial u_{-}^{\delta}}{\partial n}=\frac{\partial u_{+}^{\delta}}{\partial n} \quad \text { on } \Gamma
$$


For the inner boundary, we will consider two cases.

1. Perfectly Electric Conductor

2. Perfect Magnetic Conductor

or equivalently,

1. Dirichlet Boundary Condition

$$
u_{+}^{\delta}=0 \quad \text { on } \Gamma^{\delta}
$$

2. Neumann Boundary Condition

$$
\frac{\partial u_{+}^{\delta}}{\partial n}=0 \quad \text { on } \Gamma^{\delta}
$$

In the following generalized impedance boundary conditions (GIBC's) will be derived for both cases: We seek a boundary operator $D^{\delta}$ for Dirichlet and $N^{\delta}$ for Neumann such that (for regular coefficients and surfaces)

$$
D^{\delta}, N^{\delta}: C^{\infty}(\Gamma) \rightarrow C^{\infty}(\Gamma)
$$

and we consider $\widetilde{u}^{\delta}$ solution of

$$
\Delta \widetilde{u}^{\delta}+k_{0}^{2} \widetilde{u}^{\delta}=0 \quad \text { in } \Omega_{-}
$$

where

$$
\widetilde{u}^{\delta}=u^{i}+\widetilde{u}_{s}^{\delta} \quad \text { in } \Omega_{-},
$$

$\widetilde{u}_{s}^{\delta}$ satisfies the Sommerfeld Radiation Condition and

$$
\widetilde{u}^{\delta}+D^{\delta} \frac{\partial \widetilde{u}^{\delta}}{\partial n}=0 \quad \text { on } \Gamma
$$

in the Dirichlet case, or

$$
\frac{\partial \widetilde{u}^{\delta}}{\partial n}+N^{\delta} \widetilde{u}^{\delta}=0 \quad \text { on } \Gamma
$$

in the Neumann case, then a formal error estimate of the form

$$
\left\|u_{-}^{\delta}-\widetilde{u}^{\delta}\right\|_{\Omega_{-}} \leq c \delta^{m+1}
$$

holds for $\delta$ sufficiently small (see for instance 2]). If (13) applies then the GIBC is said to be of order m. We shall denote by $D^{\delta, m}$ and $N^{\delta, m}$ the impedance operator associated with the order $m$. 


\section{Parametric Coordinates}

Consider a $C^{2}$ curve $\Gamma$, boundary of a domain $\Omega$ (assumed to be simply connected for simplicity). We assume that $\Gamma$ is parametrized (locally) as

$$
x_{\Gamma}(t)=\left(\begin{array}{l}
x(t) \\
y(t)
\end{array}\right) ; t \in I \subset \mathbb{R}
$$

and that this parametrization defines a counter-clockwise orientation.

Let $s_{0}=\inf \frac{1}{|c(t)|}$ where $c(t)$ is the curvature and $\Omega_{0}=\{x$ such that $d(x, \Gamma) \leq$ $\left.s_{0}\right\}$, then one can associate to every $x \in \Omega_{0}$ a unique couple $\left(x_{\Gamma}, \nu\right) \in \Gamma \times$ $\left[-s_{0}, s_{0}\right]$ such that

$$
x=x_{\Gamma}(t)+\nu \vec{n}(t)
$$

for some $t \in I$ where $\vec{n}(t)$ is the inward unitary normal (see Appendix). $x_{\Gamma}$ is the orthogonal projection of $\mathrm{x}$ on $\Gamma .\left(x_{\Gamma}, \nu\right)$ denotes then the parametric coordinates associated with $x$. Since there is a bijection between the curvilinear abscissa $s(t)$ and $x_{\Gamma}(t), \mathrm{x}$ is also uniquely determined by the couple $(s, \nu) \in \mathbb{R}_{+} \times\left[-s_{0}, s_{0}\right]$ that we also shall refer to as (curvilinear or) parametric coordinates.

\section{Expression of Differential Operators in Curvilinear Coor- dinates}

Let $u$ be a function defined on $\Omega_{0}$ and $\widetilde{u}: \mathbb{R}_{+} \times\left[-s_{0}, s_{0}\right]$ by

$$
\widetilde{u}(s, \nu)=u(x)
$$

where $x=x_{\Gamma}(t)+\nu \vec{n}(t)$ and $d s(t)=\left|x_{\Gamma}^{\prime}(t)\right|$. Then, the gradient and laplacian of function $\mathrm{u}$ are given by

$$
\nabla u=\frac{1}{(1+\nu c)} \frac{\partial \widetilde{u}}{\partial s} \frac{\vec{\tau}}{\|\vec{\tau}\|}+\frac{\partial \widetilde{u}}{\partial \nu} \vec{n}
$$

where $\vec{\tau}$ is the tangential vector defined by $\vec{\tau}=\frac{d}{d t} x_{\Gamma}$ and

$$
\Delta u=\frac{1}{(1+\nu c)} \frac{\partial}{\partial s} \frac{1}{(1+\nu c)} \frac{\partial}{\partial s} \widetilde{u}+\frac{1}{(1+\nu c)} \frac{\partial}{\partial \nu}(1+\nu c) \frac{\partial}{\partial \nu} \widetilde{u}
$$

\section{Thin Coating with Variable Width}

We assume that $\Omega$ contains $\Omega^{\delta}$ whose boundary $\Gamma^{\delta}$ is defined as

$$
\Gamma^{\delta}=\left\{x_{\Gamma}^{\delta}(t)=x_{\Gamma}(t)+\delta(t) \vec{n}(t), t \in I\right\}
$$

where $\delta(t)$ is a positive function such that $0<\delta(t)<s_{0}$.

Note that, in fact we shall assume that $0<\delta(t) \leq \delta_{*}$ and the study the behavior of the solutions to a diffraction problem as $\delta_{*} \rightarrow 0$ (we shall also assume that $\left.\delta^{\prime}(t) \leq \delta_{*}\right)$ Tangential and normal vectors on $\Gamma^{\delta}$ can be expressed in the form

$$
\begin{array}{r}
\vec{\tau}^{\delta}=x_{\Gamma}^{\delta^{\prime}}=\vec{\tau}+\delta^{\prime} \vec{n}+\delta c \vec{\tau} \\
=(1+\delta c) \vec{\tau}+\delta^{\prime} \vec{n}
\end{array}
$$




$$
\vec{n}^{\delta}=\frac{1}{\left\|\overrightarrow{\tau^{\delta}}\right\|}\left((1+\delta c)\|\vec{\tau}\| \vec{n}-\delta^{\prime} \frac{\vec{\tau}}{\|\vec{\tau}\|}\right)
$$

where

$$
\left\|\vec{\tau}^{\delta}\right\|=\sqrt{(1+\delta c)^{2}\|\vec{\tau}\|+\left|\delta^{\prime}\right|^{2}}
$$

Let us assume that $\delta(t)=\delta_{0} f(t)$ with $\delta_{0} \ll 1 . \quad f(t)$ is a real single-valued function that depends on the width of the coating.

\section{Asymptotic Expansions and Derivation of the GIBC's}

$\delta(t)$ will be represented as

$$
\delta(s)=\delta_{0} f(s)
$$

If we scale as $\xi=\frac{\nu}{\delta_{0}}$ in $\Omega_{+}^{\delta}$ and seek total field in the form

$$
u_{+}^{\delta}(x)=\sum_{j=0}^{\infty} \delta_{0}^{j} u_{+}^{j}\left(s, \frac{\nu}{\delta_{0}}\right)=\widetilde{u}_{+}^{\delta}(s, \xi) \quad \text { in } \Omega_{+}^{\delta}
$$

and

$$
u_{-}^{\delta}(x)=\sum_{j=0}^{\infty} \delta_{0}^{j} u_{-}^{j}(x)=\widetilde{u}_{-}^{\delta}(x) \quad \text { in } \Omega_{-},
$$

then, the boundary value problem in Chapter 2 can be reformulated using these new notation. Respectively, $\widetilde{u}_{+}^{\delta}$ and $\widetilde{u}_{-}^{\delta}$ will denote $\widetilde{u}^{\delta}$ in $\Omega_{+}^{\delta}$ and $\Omega_{-} . u_{+}^{j}$ : $[0, L] \times[0, f(s)]$ where $L$ is the length of $\Gamma$. From (18), (24) and (25), the boundary value problem in (11)-(8) can be rewritten as

$$
\begin{gathered}
\frac{1}{\left(1+\delta_{0} \xi c\right)} \frac{\partial}{\partial s} \frac{1}{\left(1+\delta_{0} \xi c\right)} \frac{\partial \widetilde{u}_{+}^{\delta}}{\partial s}+\frac{1}{\delta_{0}^{2}} \frac{1}{\left(1+\delta_{0} \xi c\right)} \frac{\partial}{\partial \xi}\left(1+\delta_{0} \xi c\right) \frac{\partial \widetilde{u}_{+}^{\delta}}{\partial \xi}+k_{1}^{2} \widetilde{u}_{+}^{\delta}=0 \\
\widetilde{u}_{-}^{\delta}\left(x_{\Gamma}(s)\right)=\widetilde{u}_{+}^{\delta}(s, 0) \\
\frac{\partial \widetilde{u}_{-}^{\delta}\left(x_{\Gamma}(s)\right)}{\partial n}=\frac{1}{\delta_{0}} \frac{\partial \widetilde{u}_{+}^{\delta}(s, 0)}{\partial \xi} \\
\frac{\partial \widetilde{u}_{+}^{\delta}(s, f(s))}{\partial n}=0 \text { for Neumann BC }
\end{gathered}
$$

In the following from (26)-(30), impedance operators will be derived for Dirichlet and Neumann case, respectively. 


\subsection{Derivation of the GIBC for the Dirichlet case}

Substituting (24) into equation (26) and boundary conditions (28) and (29), one can obtain the following system of boundary value problems (BVP's)

$$
\begin{gathered}
\frac{\partial^{2}}{\partial \xi^{2}} u_{+}^{j+2}+\left[3 \xi c \frac{\partial^{2}}{\partial \xi^{2}}+c \frac{\partial}{\partial \xi}\right] u_{+}^{j+1}+\left[3 \xi^{2} c^{2} \frac{\partial^{2}}{\partial \xi^{2}}+2 \xi c^{2} \frac{\partial}{\partial \xi}+\frac{\partial^{2}}{\partial s^{2}}+k_{1}^{2}\right] u_{+}^{j} \\
+\left[\xi^{3} c^{3} \frac{\partial^{2}}{\partial \xi^{2}}+\xi^{2} c^{3} \frac{\partial}{\partial \xi}+\xi c \frac{\partial^{2}}{\partial s^{2}}-\xi c^{\prime} \frac{\partial}{\partial s}+3 \xi k_{1}^{2} c\right] u_{+}^{j-1}+3 \xi^{2} k_{1}^{2} c^{2} u_{+}^{j-2} \\
+\xi^{3} k_{1}^{2} c^{3} u_{+}^{j-3}=0 \\
\frac{\partial}{\partial \xi} u_{+}^{j+1}(s, 0)=\frac{\partial}{\partial n} u_{-}^{j}(s, 0)=\varphi_{j}(s) \\
u_{+}^{j}(s, f(s))=0
\end{gathered}
$$

where (26) was multiplied by $\left(1+\delta_{0} \xi c\right)$ and $\left(1+\delta_{0} \xi c\right)^{2}$, respectively. In the following the system of BVP's (31)-(33) will be solved for $u_{+}^{j}$ for $j=0,1,2,3$.

\section{Order 0:}

For $\mathrm{j}=0$, one can easily solve the BVP

$$
\begin{gathered}
\frac{\partial^{2} u_{+}^{0}}{\partial \xi^{2}}=0 \\
u_{+}^{0}(s, f(s))=0 \\
\frac{\partial u_{+}^{0}}{\partial \xi}(s, 0)=0
\end{gathered}
$$

and can find $u_{+}^{0}$, namely,

$$
u_{+}^{0}(s, \xi)=0
$$

\section{Order 1:}

When the BVP for $\mathrm{j}=1$

$$
\begin{gathered}
\frac{\partial^{2} u_{+}^{1}}{\partial \xi^{2}}=-\left(3 \xi c \frac{\partial^{2}}{\partial \xi^{2}}+c \frac{\partial}{\partial \xi}\right) u_{+}^{0} \\
u_{+}^{1}(s, 0)=0 \\
\frac{\partial u_{+}^{1}}{\partial \xi}(s, 0)=\varphi_{0}
\end{gathered}
$$

is solved, one can easily obtain

$$
u_{+}^{1}(s, \xi)=(\xi-f) \vartheta_{0}
$$




\section{Order 2:}

When the BVP for $\mathrm{j}=2$

$$
\begin{gathered}
\frac{\partial^{2} u_{+}^{2}}{\partial \xi^{2}}=-\left(3 \xi c \frac{\partial^{2}}{\partial \xi^{2}}+c \frac{\partial}{\partial \xi}\right) u_{+}^{1}-\left(3 \xi^{2} c^{2} \frac{\partial^{2}}{\partial \xi^{2}}+2 \xi c^{2} \frac{\partial}{\partial \xi}+\frac{\partial^{2}}{\partial s^{2}}+k_{1}^{2}\right) u_{+}^{0} \\
u_{+}^{2}(s, 0)=0 \\
\frac{\partial u_{+}^{2}}{\partial \xi}(s, 0)=\varphi_{1}
\end{gathered}
$$

is solved, one can easily obtain

$$
u_{+}^{2}(s, \xi)=(\xi-f) \vartheta_{1}-\frac{1}{2}\left(\xi^{2}-f^{2}\right) c \vartheta_{0}
$$

\section{Order 3:}

When the BVP for $\mathrm{j}=3$

$$
\begin{gathered}
\frac{\partial^{2} u_{+}^{3}}{\partial \xi^{2}}=-\left(3 \xi c \frac{\partial^{2}}{\partial \xi^{2}}+c \frac{\partial}{\partial \xi}\right) u_{+}^{2}-\left(3 \xi^{2} c^{2} \frac{\partial^{2}}{\partial \xi^{2}}+2 \xi c^{2} \frac{\partial}{\partial \xi}+\frac{\partial^{2}}{\partial s^{2}}+k_{1}^{2}\right) u_{+}^{1} \\
-\left(\xi^{3} c^{3} \frac{\partial^{2}}{\partial \xi^{2}}+\xi^{2} c^{3} \frac{\partial}{\partial \xi}+\xi c \frac{\partial^{2}}{\partial s^{2}}-\xi c^{\prime} \frac{\partial}{\partial s}+3 \xi c k_{1}^{2}\right) u_{+}^{0} \\
u_{+}^{3}(s, 0)=0 \\
\frac{\partial u_{+}^{3}}{\partial \xi}(s, 0)=\varphi_{2}
\end{gathered}
$$

is solved, one can easily obtain

$$
\begin{array}{r}
u_{+}^{3}(s, \xi)=(\xi-f) \vartheta_{2}-\frac{1}{2}\left(\xi^{2}-f^{2}\right) \vartheta_{1} c+\frac{1}{3}\left(\xi^{3}-f^{3}\right) c^{2} \vartheta_{0} \\
-\frac{1}{6}\left(\xi^{3}-f^{3}\right)\left(\frac{\partial^{2} \vartheta_{0}}{\partial s^{2}}+k_{1}^{2} \vartheta_{0}\right) \\
+\frac{1}{2}\left(\xi^{2}-f^{2}\right)\left(\frac{\partial^{2}}{\partial s^{2}}\left(f \vartheta_{0}\right)+k_{1}^{2} f \vartheta_{0}\right)
\end{array}
$$

GIBC's For Dirichlet:

Dirichlet Boundary Condition (11) can be denoted as

$$
\widetilde{u}_{-}^{\delta, k}\left(x_{\Gamma}(s)\right)=-D^{\delta, k} \frac{\partial \widetilde{u}_{-}^{\delta, k}\left(x_{\Gamma}(s)\right)}{\partial n}
$$

where $\mathrm{k}$ is order of impedance operator. Substituting (24) and (25) into (38), one can obtain

$$
\sum_{j=0}^{k} \delta^{j} u_{-}^{j}\left(x_{\Gamma}(s)\right)=-D^{\delta, k}\left(\sum_{j=0}^{k} \delta^{j} \frac{\partial u_{-}^{j}\left(x_{\Gamma}(s)\right)}{\partial n}\right)
$$


Similarly, substituting (27) and (32) into (39), one can obtain

$$
\sum_{j=0}^{k} \delta^{j} u_{+}^{j}(s, 0)=-D^{\delta, k}\left(\sum_{j=0}^{k} \delta^{j} \varphi_{j}\right)
$$

Respectively, for $\mathrm{k}=0,1,2,3$ substituting (34)-(37) into (40), GIBC's for the Dirichlet case can be obtained as

$$
\begin{gathered}
D^{\delta, 0}=0 \\
D^{\delta, 1}=\delta(s) \\
D^{\delta, 2}=\delta(s)\left(1-\frac{1}{2} \delta(s) c(s)\right) \\
D^{\delta, 3}=\delta(s)\left(1-\frac{1}{2} \delta(s) c(s)+\frac{1}{3} \delta^{2}(s) c^{2}(s)\right)-\frac{1}{6} \delta^{3}(s)\left(\frac{\partial^{2}}{\partial s^{2}}+k_{1}^{2}\right) \\
+\frac{1}{2} \delta^{2}(s)\left(\frac{\partial^{2}}{\partial s^{2}}+k_{1}^{2}\right) \delta(s)
\end{gathered}
$$

\section{Derivation of the GIBC for the Neumann case}

Analogously, GIBC's for the Neumann case will be derived. Reduced wave equation (31) has already been obtained by depending index j. From gradient of function $\mathrm{u}$ given in (17), Neumann Boundary Condition can be expressed as

$$
\left(\frac{\partial \widetilde{u}_{+}^{\delta}(s, f(s))}{\partial s} \frac{\vec{\tau}}{\|\vec{\tau}\|}+\left(1+\delta_{0} f c\right) \frac{1}{\delta} \frac{\partial \widetilde{u}_{+}^{\delta}(s, f(s))}{\partial \xi} \vec{n}\right) \cdot \vec{n}^{\delta}=0
$$

Substituting $\vec{n}^{\delta}$ given in (21) into (45) and rearranging the expression, one can obtain

$$
\frac{\partial \widetilde{u}_{+}^{\delta}(s, f(s))}{\partial \xi}=\delta_{0} \frac{\delta^{\prime}}{\|\vec{\tau}\|} \frac{1}{(1+\delta c)^{2}} \frac{\partial \widetilde{u}_{+}^{\delta}(s, f(s))}{\partial s}
$$

Expanding Maclaurin series for $1 /(1+\delta c)^{2}$, (46) can be rewritten as

$$
\frac{\partial \widetilde{u}_{+}^{\delta}(s, f(s))}{\partial \xi}=\delta_{0} \frac{\delta^{\prime}}{\|\vec{\tau}\|}\left(1-2 \delta c+3 \delta^{2} c^{2}\right) \frac{\partial \widetilde{u}_{+}^{\delta}(s, f(s))}{\partial s}+O\left(\delta^{5}\right)
$$

Note that,

$$
\frac{f^{\prime}}{\|\vec{\tau}\|}=\frac{\partial f}{\partial s}
$$


and from now on

$$
\frac{\partial f}{\partial s}=f^{\prime}
$$

and

$$
\frac{\partial \delta}{\partial s}=\delta^{\prime}
$$

are used as new notation.

Substituting series given in (24) and (25) into (27) and (47) and considering (31), one can obtain the following system of BVP's:

$$
\begin{gathered}
\frac{\partial^{2}}{\partial \xi^{2}} u_{+}^{j+2}+\left[3 \xi c \frac{\partial^{2}}{\partial \xi^{2}}+c \frac{\partial}{\partial \xi}\right] u_{+}^{j+1}+\left[3 \xi^{2} c^{2} \frac{\partial^{2}}{\partial \xi^{2}}+2 \xi c^{2} \frac{\partial}{\partial \xi}+\frac{\partial^{2}}{\partial s^{2}}+k_{1}^{2}\right] u_{+}^{j} \\
+\left[\xi^{3} c^{3} \frac{\partial^{2}}{\partial \xi^{2}}+\xi^{2} c^{3} \frac{\partial}{\partial \xi}+\xi c \frac{\partial^{2}}{\partial s^{2}}-\xi c^{\prime} \frac{\partial}{\partial s}+3 \xi k_{1}^{2} c\right] u_{+}^{j-1}+3 \xi^{2} k_{1}^{2} c^{2} u_{+}^{j-2} \\
+\xi^{3} k_{1}^{2} c^{3} u_{+}^{j-3}=0 \\
\frac{\partial u_{+}^{j+2}(s, f)}{\partial \xi}=f^{\prime}\left(\frac{\partial u_{+}^{j}(s, f)}{\partial s}-2 f c \frac{\partial u_{+}^{j-1}(s, f)}{\partial s}+3 f^{2} c^{2} \frac{\partial u_{+}^{j-2}(s, f)}{\partial s}\right) \\
u_{+}^{j}(s, 0)=\Psi_{j}
\end{gathered}
$$

System of BVP's (48)- (50) should be solved to obtain total fields $\frac{\partial u_{+}^{j}}{\partial \xi}(\mathrm{j}=0$, $1,2,3,4)$. Thus, one can derive GIBC's for the Neumann case. In the following system of BVP's (48)-(50) and their solutions will be expressed for $\mathrm{j}=0,1,2$, 3,4 , respectively.

\section{Order 0:}

If the BVP for $\mathrm{j}=0$

$$
\begin{gathered}
\frac{\partial^{2} u_{+}^{0}}{\partial \xi^{2}}=0 \\
\frac{\partial u_{+}^{0}}{\partial \xi}(s, f(s))=0 \\
u_{+}^{0}(s, 0)=\Psi_{0}
\end{gathered}
$$

is solved, one can obtain

$$
u_{+}^{0}(s, \xi)=\Psi_{0}
$$

Thus, derivative of $u_{+}^{0}$ is

$$
\frac{\partial u_{+}^{0}(s, \xi)}{\partial \xi}=0
$$




\section{Order 1:}

If the BVP for $\mathrm{j}=1$

$$
\begin{gathered}
\frac{\partial^{2} u_{+}^{1}}{\partial \xi^{2}}=-\left(3 \xi c \frac{\partial^{2}}{\partial \xi^{2}}+c \frac{\partial}{\partial \xi}\right) u_{+}^{0} \\
\frac{\partial u_{+}^{1}}{\partial \xi}(s, f(s))=0 \\
u_{+}^{1}(s, 0)=\Psi_{1}
\end{gathered}
$$

is solved, one can obtain

$$
u_{+}^{1}(s, \xi)=\Psi_{1}
$$

Thus, derivative of $u_{+}^{1}$ is

$$
\frac{\partial u_{+}^{1}(s, \xi)}{\partial \xi}=0
$$

\section{Order 2:}

If the BVP for $\mathrm{j}=2$

$$
\begin{gathered}
\frac{\partial^{2} u_{+}^{2}}{\partial \xi^{2}}=-\left(3 \xi c \frac{\partial^{2}}{\partial \xi^{2}}+c \frac{\partial}{\partial \xi}\right) u_{+}^{1}-\left(3 \xi^{2} c^{2} \frac{\partial^{2}}{\partial \xi^{2}}+2 \xi c^{2} \frac{\partial}{\partial \xi}+\frac{\partial^{2}}{\partial s^{2}}+k_{1}^{2}\right) u_{+}^{0} \\
\frac{\partial u_{+}^{2}}{\partial \xi}=f^{\prime} \frac{\partial u_{+}^{0}}{\partial s} \\
u_{+}^{2}(s, 0)=\Psi_{2}
\end{gathered}
$$

is solved, one can obtain

$$
u_{+}^{2}(s, \xi)=\Psi_{2}+\xi f^{\prime} \frac{\partial}{\partial s} \Psi_{0}-\left(\frac{1}{2} \xi^{2}-f \xi\right)\left(\frac{\partial^{2}}{\partial s^{2}}+k_{1}^{2}\right) \Psi_{0}
$$

Thus, derivative of $u_{+}^{2}$ is

$$
\frac{\partial u_{+}^{2}}{\partial \xi}=f^{\prime} \frac{\partial}{\partial s} \Psi_{0}-(\xi-f)\left(\frac{\partial^{2}}{\partial s^{2}}+k_{1}^{2}\right) \Psi_{0}
$$

\section{Order 3:}

If the BVP for $\mathrm{j}=3$

$$
\begin{array}{r}
\frac{\partial^{2} u_{+}^{3}}{\partial \xi^{2}}=-\left(3 \xi c \frac{\partial^{2}}{\partial \xi^{2}}+c \frac{\partial}{\partial \xi}\right) u_{+}^{2}-\left(3 \xi^{2} c^{2} \frac{\partial^{2}}{\partial \xi^{2}}+2 \xi c^{2} \frac{\partial}{\partial \xi}+\frac{\partial^{2}}{\partial s^{2}}+k_{1}^{2}\right) u_{+}^{1} \\
-\left(\xi^{3} c^{3} \frac{\partial^{2}}{\partial \xi^{2}}+\xi^{2} c^{3} \frac{\partial}{\partial \xi}+\xi c \frac{\partial^{2}}{\partial s^{2}}-\xi c^{\prime} \frac{\partial}{\partial s}+3 \xi c k_{1}^{2}\right) u_{+}^{0}
\end{array}
$$




$$
\begin{gathered}
\frac{\partial u_{+}^{3}}{\partial \xi}(s, f(s))=f^{\prime}\left(\frac{\partial u_{+}^{1}}{\partial s}-2 f c \frac{\partial u_{+}^{0}}{\partial s}\right) \\
u_{+}^{3}(s, 0)=\Psi_{3}
\end{gathered}
$$

is solved, one can obtain

$$
\begin{aligned}
\frac{\partial u_{+}^{3}}{\partial \xi}=f^{\prime} \frac{\partial}{\partial s} \Psi_{1}- & 2 f f^{\prime} c \frac{\partial}{\partial s} \Psi_{0}+\frac{1}{2}\left(\xi^{2}-f^{2}\right)\left(3 c \frac{\partial^{2}}{\partial s^{2}}+c^{\prime} \frac{\partial}{\partial s}+c k_{1}^{2}\right) \Psi_{0} \\
- & (\xi-f)\left(c\left(\frac{\partial}{\partial s} f \frac{\partial}{\partial s}+f k_{1}^{2}\right) \Psi_{0}+\left(\frac{\partial^{2}}{\partial s^{2}}+k_{1}^{2}\right) \Psi_{1}\right)
\end{aligned}
$$

\section{Order 4:}

If the BVP for $\mathrm{j}=4$

$$
\begin{gathered}
\frac{\partial^{2} u_{+}^{4}}{\partial \xi^{2}}=-\left(3 \xi c \frac{\partial^{2}}{\partial \xi^{2}}+c \frac{\partial}{\partial \xi}\right) u_{+}^{3}-\left(3 \xi^{2} c^{2} \frac{\partial^{2}}{\partial \xi^{2}}+2 \xi c^{2} \frac{\partial}{\partial \xi}+\frac{\partial^{2}}{\partial s^{2}}+k_{1}^{2}\right) u_{+}^{2} \\
-\left(\xi^{3} c^{3} \frac{\partial^{2}}{\partial \xi^{2}}+\xi^{2} c^{3} \frac{\partial}{\partial \xi}+\xi c \frac{\partial^{2}}{\partial s^{2}}-\xi c^{\prime} \frac{\partial}{\partial s}+3 \xi c k_{1}^{2}\right) u_{+}^{1}-3 \xi^{2} c^{2} k_{1}^{2} u_{+}^{0} \\
\frac{\partial u_{+}^{4}}{\partial \xi}(s, f(s))=f^{\prime}\left(\frac{\partial u_{+}^{2}}{\partial s}-2 f c \frac{\partial u_{+}^{1}}{\partial \xi}+3 f^{2} c^{2} \frac{\partial u_{+}^{0}}{\partial s}\right) \\
u_{+}^{4}(s, 0)=\Psi_{4}
\end{gathered}
$$

is solved, one can obtain

$$
\begin{array}{r}
\frac{\partial u_{+}^{4}}{\partial \xi}=f^{\prime}\left[f \frac{\partial}{\partial s} f^{\prime} \frac{\partial}{\partial s}+f \frac{\partial}{\partial s} f A-\frac{1}{2} f^{2} \frac{\partial}{\partial s} A+3 f^{2} c^{2} \frac{\partial}{\partial s}\right] \Psi_{0}-2 f f^{\prime} c \frac{\partial}{\partial s} \Psi_{1} \\
+f^{\prime} \frac{\partial}{\partial s} \Psi_{2}+\frac{1}{3}\left(\xi^{3}-f^{3}\right)\left[\left(-\frac{7}{2} c A_{0}+5 c^{2} A+\frac{1}{2} A A-3 c^{2} k_{1}^{2}\right) \Psi_{0}\right] \\
+\frac{1}{2}\left(\xi^{2}-f^{2}\right)\left[\left(4 c A-A_{2}\right) \Psi_{1}+\left(4 c^{2} A_{1}-\left(2 c^{2}+A\right)\left(f^{\prime} \frac{\partial}{\partial s}+f A\right)\right) \Psi_{0}\right] \\
+(\xi-f) \\
{\left[-A \Psi_{2}-c\left(f^{\prime} \frac{\partial}{\partial s}+f A\right) \Psi_{1}+\left(2 f f^{\prime} c^{2} \frac{\partial}{\partial s}+\frac{1}{2} f^{2} c A_{0}-f c^{2} A_{1}\right) \Psi_{0}\right]}
\end{array}
$$

where

$$
\begin{gathered}
A=\frac{\partial^{2}}{\partial s^{2}}+k_{1}^{2} \\
A_{0}=3 c \frac{\partial^{2}}{\partial s^{2}}+c^{\prime} \frac{\partial}{\partial s}+c k_{1}^{2} \\
A_{1}=\frac{\partial}{\partial s} f \frac{\partial}{\partial s}+f k_{1}^{2} \\
A_{2}=c \frac{\partial^{2}}{\partial s^{2}}-c^{\prime} \frac{\partial}{\partial s}+3 c k_{1}^{2}
\end{gathered}
$$


GIBC's for Neumann:

Neumann Boundary Condition (12) ) can be denoted as

$$
\frac{\partial \widetilde{u}_{-}^{\delta, k}\left(x_{\Gamma}(s)\right)}{\partial n}=-N^{\delta, k}\left(\widetilde{u}_{-}^{\delta, k}\left(x_{\Gamma}(s)\right)\right)
$$

where $\mathrm{k}$ is order of impedance operator. Substituting (24) and (25) into (56), one can obtain

$$
\sum_{j=0}^{k} \delta^{j} \frac{\partial u_{-}^{j}\left(x_{\Gamma}(s)\right)}{\partial n}=-N^{\delta, k}\left(\sum_{j=0}^{k} \delta^{j} u_{-}^{j}\left(x_{\Gamma}(s)\right)\right)
$$

Similarly, substituting (27), (32) and (50) into (157), one can obtain

$$
\sum_{j=0}^{k} \delta^{j} \varphi_{j}=-N^{\delta, k}\left(\sum_{j=0}^{k} \delta^{j} \Psi_{j}\right)
$$

Considering (32), from (51)-(55), expressions of $\varphi_{j}(\mathrm{k}=0,1,2,3,4)$ can be obtained. Substituting $\varphi_{j}$ 's $(\mathrm{k}=0,1,2,3,4)$ into (58), GIBC's for the Neumann case can be obtained as

$$
\begin{gathered}
N^{\delta, 0}=0 \\
N^{\delta, 1}=-\left(\frac{\partial}{\partial s} \delta(s) \frac{\partial}{\partial s}+\delta(s) k_{1}^{2}\right) \\
N^{\delta, 2}=-\frac{\partial}{\partial s} \delta(s) \frac{\partial}{\partial s}+\frac{1}{2} \frac{\partial}{\partial s} \delta^{2}(s) c(s) \frac{\partial}{\partial s}-k_{1}^{2}\left(\delta(s)+\frac{1}{2} \delta^{2}(s) c(s)\right) \\
N^{\delta, 3}=-k_{1}^{2} \delta(s)\left(1+\frac{1}{2} \delta(s) c(s)+\frac{1}{3} \delta^{2}(s) k_{1}^{2}+\frac{1}{2} \delta(s) \delta^{\prime \prime}(s)+\left(\delta^{\prime}(s)\right)^{2}\right) \\
-\frac{\partial}{\partial s} \delta(s) \frac{1}{\partial s}+\frac{\partial}{2} \delta^{2}(s) c(s) \frac{\partial}{\partial s}-\frac{1}{3} \frac{\partial}{\partial s} \delta^{3}(s) c^{2}(s) \frac{\partial}{\partial s}-\frac{2}{3} k_{1}^{2} \frac{\partial}{\partial s} \delta^{3}(s) \frac{\partial}{\partial s} \\
-\frac{1}{3} \frac{\partial^{2}}{\partial s^{2}} \delta^{3}(s) \frac{\partial^{2}}{\partial s^{2}}-\frac{1}{2} \frac{\partial}{\partial s} \delta^{2}(s) \delta^{\prime \prime}(s) \frac{\partial}{\partial s}
\end{gathered}
$$

\section{Numerical Method - Finite Elements Method}

In previous sections, GIBC's for the Dirichlet case and Neumann have been derived. In this section, the boundary value problems (10)-(8) are solved numerically for exact and approximate solutions. The Finite Elements Method (FEM) is selected to solve the problems numerically. In the following FEM is constructed for the cases of exact and approximate solutions, respectively. 


\subsection{The Exact Solution}

Consider Figure 1 and the boundary value problems (11)-(8). Firstly, it is assumed that the regions $\Omega_{-}$and $\Omega_{+}^{\delta}$ are meshed as triangulation. In order to bound the domain of calculations, the boundary condition

$$
\frac{\partial u^{s}}{\partial \bar{n}}-\left.i k_{0} u^{s}\right|_{\Gamma_{R}}=0
$$

is selected as an approximation of the Sommerfeld Radiation Condition, where $\bar{n}$ is a outward unit normal vector and $\Gamma_{R}$ is a circle with radius $R$, chosen to be sufficiently large. The variational formulation is obtained by multiplying (1)-(2) by $\bar{\nu}$ and integrating the results over $\Omega_{-}$and $\Omega_{+}^{\delta}$, namely,

$$
\int_{\Omega_{-}} \Delta u_{-}^{\delta} \bar{\nu}+\int_{\Omega_{+}^{\delta}} \Delta u_{+}^{\delta} \bar{\nu}+k_{0}^{2} \int_{\Omega_{-}} u_{-}^{\delta} \bar{\nu}+k_{1}^{2} \int_{\Omega_{+}^{\delta}} u_{+}^{\delta} \bar{\nu}=0
$$

where $\bar{\nu}$ is a test function. Then considering Neumann or Dirichlet Boundary Condition, by Green's formula, the problem is converted into finding $u_{-}^{\delta}$ and $u_{+}^{\delta}$ such that

$$
\begin{array}{r}
\int_{\Omega_{-}} \nabla u_{-}^{\delta} \nabla \bar{\nu}+\int_{\Omega_{+}^{\delta}} \nabla u_{+}^{\delta} \nabla \bar{\nu}-k_{0}^{2} \int_{\Omega_{-}} u_{-}^{\delta} \bar{\nu}-k_{1}^{2} \int_{\Omega_{+}^{\delta}} u_{+}^{\delta} \bar{\nu} \\
-\int_{\Gamma_{R}} i k_{0} u_{-}^{\delta} \bar{\nu} d s-\int_{\Gamma_{R}}\left(\frac{\partial u^{i}}{\partial \bar{n}}-i k_{0} u^{i}\right) \bar{\nu} d s=0
\end{array}
$$

Note that, 65 already contains Neumann Boundary Condition while not contains Dirichlet Boundary Condition. Thus, $u_{+}^{\delta}(s, f(s))=0$ must be taken into consideration for the Dirichlet case. Also, $\bar{\nu}(s, f(s))=0$ for the Dirichlet case. We seek unknown functions $u_{-}^{\delta}, u_{+}^{\delta}$ and test function $\bar{\nu}$ in serial form as

$$
\sum_{k=0}^{M} w_{k} \phi_{k}(x, y)
$$

where $w_{k}$ 's are unknown real coefficient, $\phi_{k}$ 's are known hat functions which are continuous piecewise affine and are equal to 1 on one vertex and 0 on all others. $M$ is the number of vertices. Substituting series (66) into the variational formula (65), a system of linear equation is obtained. If one solves the system of linear equation, the total field is obtained in whole space.

\subsection{The Approximate Solution For the Dirichlet case}

Executing FEM, the approximate solution is solved for the Dirichlet case as in the previous section. This time, only the region $\Omega_{-}$is meshed. Thus, executing the same procedure as in the previous section, one can obtain

$$
\begin{array}{r}
\int_{\Omega_{-}} \nabla u_{-}^{\delta} \nabla \bar{\nu}-k_{0}^{2} \int_{\Omega_{-}} u_{-}^{\delta} \bar{\nu}-\int_{\Gamma} \frac{\partial u}{\partial n} \bar{\nu} d s-\int_{\Gamma_{R}} i k_{0} u_{-}^{\delta} \bar{\nu} d s \\
-\int_{\Gamma_{R}}\left(\frac{\partial u^{i}}{\partial \bar{n}}-i k_{0} u^{i}\right) \bar{\nu} d s=0
\end{array}
$$

In the following the variational formulations are derived for order 1,2 and 3, respectively. 
The variational formulation For $D^{\delta, 1}$ :

If one substitute (42) into (11) and rearrange the result,

$$
\frac{\partial u_{-}^{\delta}}{\partial n}=-\frac{1}{\delta(s)} u_{-}^{\delta} \quad \text { on } \Gamma
$$

is obtained. Substituting (68) into (67), the variational formulation

$$
\begin{aligned}
\int_{\Omega_{-}} \nabla u_{-}^{\delta} \nabla \bar{\nu}-k_{0}^{2} \int_{\Omega_{-}} u_{-}^{\delta} \bar{\nu}+ & \int_{\Gamma} \frac{1}{\delta(s)} u_{-}^{\delta} \bar{\nu} d s-\int_{\Gamma_{R}} i k_{0} u_{-}^{\delta} \bar{\nu} d s \\
& -\int_{\Gamma_{R}}\left(\frac{\partial u^{i}}{\partial \bar{n}}-i k_{0} u^{i}\right) \bar{\nu} d s=0
\end{aligned}
$$

is obtained.

\section{The variational formulation For $D^{\delta, 2}$ :}

If one substitute (43) into (11) and rearrange the result,

$$
\frac{\partial u_{-}^{\delta}}{\partial n}=-\frac{1}{\delta(s)\left(1-\frac{1}{2} \delta(s) c(s)\right)} u_{-}^{\delta} \quad \text { on } \Gamma
$$

is obtained. Substituting (70) into (67), the variational formulation

$$
\begin{aligned}
\int_{\Omega_{-}} \nabla u_{-}^{\delta} \nabla \bar{\nu}- & k_{0}^{2} \int_{\Omega_{-}} u_{-}^{\delta} \bar{\nu}+\int_{\Gamma} \frac{1}{\delta(s)\left(1-\frac{1}{2} \delta(s) c(s)\right)} u_{-}^{\delta} \bar{\nu} d s \\
& -\int_{\Gamma_{R}} i k_{0} u_{-}^{\delta} \bar{\nu} d s-\int_{\Gamma_{R}}\left(\frac{\partial u^{i}}{\partial \bar{n}}-i k_{0} u^{i}\right) \bar{\nu} d s=0
\end{aligned}
$$

is obtained.

\section{The variational formulation For $D^{\delta, 3}$ :}

For order 3 Dirichlet operator has tangential derivatives of order greater than 2. Therefore, additional unknowns need to be used to set up a variational formulation that where only second tangential derivatives are present. This will result into a couple of variational formulation that will be solved together. (67) is the first variational formulation. If a new variable is selected as

$$
\psi=\frac{\partial u_{-}^{\delta}}{\partial n}
$$

(67) is converted a new form as

$$
\begin{array}{r}
\int_{\Omega_{-}} \nabla u_{-}^{\delta} \nabla \bar{\nu}-k_{0}^{2} \int_{\Omega_{-}} u_{-}^{\delta} \bar{\nu}-\int_{\Gamma} \psi \bar{\nu} d s-\int_{\Gamma_{R}} i k_{0} u_{-}^{\delta} \bar{\nu} d s \\
-\int_{\Gamma_{R}}\left(\frac{\partial u^{i}}{\partial \bar{n}}-i k_{0} u^{i}\right) \bar{\nu} d s=0
\end{array}
$$

The second variational formulation is derived by substituting (44) into (11), multiplying the result by $\bar{\nu}_{2}$ and integrating the last results over $\Gamma$, namely,

$$
\int_{\Gamma} u_{-}^{\delta} \bar{\nu}_{2} d s+\int_{\Gamma} \delta^{2}(s) \delta^{\prime}(s) \frac{\partial \psi}{\partial s} \bar{\nu}_{2} d s+\int_{\Gamma} \frac{1}{3} \delta^{3}(s) \frac{\partial^{2} \psi}{\partial s^{2}} \bar{\nu}_{2} d s+\int_{\Gamma} \alpha \psi \bar{\nu}_{2} d s=0
$$


where $\bar{\nu}_{2}$ is a test function and

$$
\alpha=\delta(s)\left(1-\frac{1}{2} \delta(s) c(s)+\frac{1}{3} \delta^{2}(s) c^{2}(s)\right)+\frac{1}{3} \delta^{3}(s) k_{1}^{2}+\frac{1}{2} \delta^{2}(s) \delta^{\prime \prime}(s)
$$

The variational formulation (74) is a strong form. To convert it in weak form, second derivatives of unknown functions $\left(\frac{\partial^{2} \psi}{\partial s^{2}}\right)$ must be vanished. By partial integration of $\int_{\Gamma} \frac{1}{3} \delta^{3}(s) \frac{\partial^{2} \psi}{\partial s^{2}} \bar{\nu}_{2} d s$, (74) can be denotes as

$$
\int_{\Gamma} u_{-}^{\delta} \bar{\nu}_{2} d s+\int_{\Gamma} \alpha \psi \bar{\nu}_{2} d s-\int_{\Gamma} \frac{1}{3} \delta^{3}(s) \frac{\partial \psi}{\partial s} \frac{\partial \bar{\nu}_{2}}{\partial s} d s=0
$$

(73) and (75) are variational formulations for order 3 .

\subsection{The Approximate Solution For the Neumann case}

Following the same procedure as in the section 4.2 , one can obtain

$$
\begin{array}{r}
\int_{\Omega_{-}} \nabla u_{-}^{\delta} \nabla \bar{\nu}-k_{0}^{2} \int_{\Omega_{-}} u_{-}^{\delta} \bar{\nu} \\
-\int_{\Gamma} \frac{\partial u}{\partial n} \bar{\nu} d s-\int_{\Gamma_{R}} i k_{0} u_{-}^{\delta} \bar{\nu} d s \\
-\int_{\Gamma_{R}}\left(\frac{\partial u^{i}}{\partial \bar{n}}-i k_{0} u^{i}\right) \bar{\nu} d s=0
\end{array}
$$

similar to (67). In the following the variational formulations are derived for order 1,2 and 3 , respectively.

The variational formulation For $N^{\delta, 1}$ :

If one substitute (60) into (12) and rearrange the result,

$$
\frac{\partial u_{-}^{\delta}}{\partial n}=\left(\frac{\partial}{\partial s} \delta(s) \frac{\partial}{\partial s}+\delta(s) k_{1}^{2}\right) u_{-}^{\delta} \quad \text { on } \Gamma
$$

is obtained. Substituting (77) into (76), the strong form of variational formulation

$$
\begin{gathered}
\int_{\Omega_{-}} \nabla u_{-}^{\delta} \nabla \bar{\nu}-k_{0}^{2} \int_{\Omega_{-}} u_{-}^{\delta} \bar{\nu}-\int_{\Gamma} \delta(s) k_{1}^{2} u_{-}^{\delta} \bar{\nu} d s-\int_{\Gamma} \delta^{\prime}(s) \frac{\partial u_{-}^{\delta}}{\partial s} \bar{\nu} d s \\
-\int_{\Gamma} \delta(s) \frac{\partial^{2} u_{-}^{\delta}}{\partial s^{2}} \bar{\nu} d s-\int_{\Gamma_{R}} i k_{0} u_{-}^{\delta} \bar{\nu} d s-\int_{\Gamma_{R}}\left(\frac{\partial u^{i}}{\partial \bar{n}}-i k_{0} u^{i}\right) \bar{\nu} d s=0
\end{gathered}
$$

is obtained. By partial integration of $\int_{\Gamma} \delta(s) \frac{\partial^{2} u_{-}^{\delta}}{\partial s^{2}} \bar{\nu} d s$, (178) can be converted into a weak form as

$$
\begin{array}{r}
\int_{\Omega_{-}} \nabla u_{-}^{\delta} \nabla \bar{\nu}-k_{0}^{2} \int_{\Omega_{-}} u_{-}^{\delta} \bar{\nu}-\int_{\Gamma} \delta(s) k_{1}^{2} u_{-}^{\delta} \bar{\nu} d s+\int_{\Gamma} \delta(s) \frac{\partial u_{-}^{\delta}}{\partial s} \frac{\partial \bar{\nu}}{\partial s} d s \\
-\int_{\Gamma_{R}} i k_{0} u_{-}^{\delta} \bar{\nu} d s-\int_{\Gamma_{R}}\left(\frac{\partial u^{i}}{\partial \bar{n}}-i k_{0} u^{i}\right) \bar{\nu} d s=0
\end{array}
$$


The variational formulation For $N^{\delta, 2}$ :

If one substitute (61) into (12) and rearrange the result,

$$
\frac{\partial u_{-}^{\delta}}{\partial n}=\left(\frac{\partial}{\partial s} \delta(s) \frac{\partial}{\partial s}-\frac{1}{2} \frac{\partial}{\partial s} \delta^{2}(s) c(s) \frac{\partial}{\partial s}+k_{1}^{2}\left(\delta(s)+\frac{1}{2} \delta^{2}(s) c(s)\right)\right) u_{-}^{\delta}
$$

is obtained.Substituting (80) into (76), the strong form of variational formulation

$$
\begin{array}{r}
\int_{\Omega_{-}} \nabla u_{-}^{\delta} \nabla \bar{\nu}-k_{0}^{2} \int_{\Omega_{-}} u_{-}^{\delta} \bar{\nu}-\int_{\Gamma} k_{1}^{2}\left(\delta(s)+\frac{1}{2} \delta^{2}(s) c(s)\right) u_{-}^{\delta} \bar{\nu} d s \\
-\int_{\Gamma}\left(\delta^{\prime}(s)-\delta(s) \delta^{\prime}(s) c(s)-\frac{1}{2} \delta^{2}(s) c^{\prime}(s)\right) \frac{\partial u_{-}^{\delta}}{\partial s} \bar{\nu} d s \\
-\int_{\Gamma}\left(\delta(s)-\frac{1}{2} \delta^{2}(s) c(s)\right) \frac{\partial^{2} u_{-}^{\delta}}{\partial s^{2}} \bar{\nu} d s \\
-\int_{\Gamma_{R}} i k_{0} u_{-}^{\delta} \bar{\nu} d s-\int_{\Gamma_{R}}\left(\frac{\partial u^{i}}{\partial \bar{n}}-i k_{0} u^{i}\right) \bar{\nu} d s=0
\end{array}
$$

is obtained, and converting the result into a weak form,

$$
\begin{array}{r}
\int_{\Omega_{-}} \nabla u_{-}^{\delta} \nabla \bar{\nu}-k_{0}^{2} \int_{\Omega_{-}} u_{-}^{\delta} \bar{\nu}+\int_{\Gamma} \alpha u_{-}^{\delta} \bar{\nu} d s+\int_{\Gamma} \gamma \frac{\partial u_{-}^{\delta}}{\partial s} \frac{\partial \bar{\nu}}{\partial s} d s \\
-\int_{\Gamma_{R}} i k_{0} u_{-}^{\delta} \bar{\nu} d s-\int_{\Gamma_{R}}\left(\frac{\partial u^{i}}{\partial \bar{n}}-i k_{0} u^{i}\right) \bar{\nu} d s=0
\end{array}
$$

is obtained where

$$
\begin{gathered}
\alpha=-k_{1}^{2}\left(\delta(s)+\frac{1}{2} c(s) \delta^{2}(s)\right) \\
\gamma=\delta(s)-\frac{1}{2} \delta^{2}(s) c(s)
\end{gathered}
$$

The variational formulation For $N^{\delta, 3}$ :

If one substitute (62) and (12) into (76) and rearrange the result, the strong form of variational formulation is obtained. If a new variable is selected as

$$
\psi=\frac{\partial^{2} u_{-}^{\delta}}{\partial s^{2}}
$$

and substitute it into the strong form of variational formulation, then vanish the expression included $\frac{\partial^{2} u_{-}^{\delta}}{\partial s^{2}}$ by partial integrations, the first variational formulation

$$
\begin{array}{r}
\int_{\Omega_{-}} \nabla u_{-}^{\delta} \nabla \bar{\nu}-k_{0}^{2} \int_{\Omega_{-}} u_{-}^{\delta} \bar{\nu}+\int_{\Gamma} \alpha u_{-}^{\delta} \bar{\nu} d s+\int_{\Gamma} \gamma \frac{\partial u_{-}^{\delta}}{\partial s} \frac{\partial \bar{\nu}}{\partial s} d s \\
-\int_{\Gamma}\left(2 \delta(s)\left(\delta^{\prime}(s)\right)^{2}+\delta^{2}(s) \delta^{\prime \prime}(s)\right) \psi \bar{\nu} d s-\int_{\Gamma} \delta^{2}(s) \delta^{\prime}(s) \frac{\partial \psi}{\partial s} \bar{\nu} d s \\
+\int_{\Gamma} \frac{1}{3} \delta^{3}(s) \frac{\partial \psi}{\partial s} \frac{\partial \bar{\nu}}{\partial s} d s-\int_{\Gamma_{R}} i k_{0} u_{-}^{\delta} \bar{\nu} d s-\int_{\Gamma_{R}}\left(\frac{\partial u^{i}}{\partial \bar{n}}-i k_{0} u^{i}\right) \bar{\nu} d s=0
\end{array}
$$


is obtained where

$$
\begin{aligned}
& \alpha=-k_{1}^{2} \delta(s)\left(1+\frac{1}{2} \delta(s)\left(c(s)+\delta^{\prime \prime}(s)\right)+\frac{1}{3} \delta^{2}(s) k_{1}^{2}+\left(\delta^{\prime}(s)\right)^{2}\right) \\
& \gamma=\delta(s)-\frac{1}{2} \delta^{2}(s) c(s)+\frac{1}{3} \delta^{3}(s) c^{2}(s)+\frac{2}{3} \delta^{3}(s) k_{1}^{2}+\frac{1}{2} \delta^{2}(s) \delta^{\prime \prime}(s)
\end{aligned}
$$

The second variational formulation is derived by multiplying (83) by $\bar{\nu}_{2}$ and integrating the results over $\Gamma$, namely,

$$
\int_{\Gamma} \frac{\partial^{2} u_{-}^{\delta}}{\partial s^{2}} \bar{\nu}_{2} d s-\int_{\Gamma} \psi \bar{\nu}_{2} d s=0
$$

Converting (85) into weak form, the second variational formulation

$$
\int_{\Gamma} \frac{\partial u_{-}^{\delta}}{\partial s} \frac{\partial \bar{\nu}_{2}}{\partial s} d s+\int_{\Gamma} \psi \bar{\nu}_{2} d s=0
$$

is obtained. (84) and (86) are variational formulations for order 3 .

\section{$5 \quad$ Numerical Results}

In this section, the numerical solutions of the problem is given to verify the accuracy and effectiveness of the GIBC's. In all cases, the coated object is assumed to be located in free-space. The errors are calculated using the error function

$$
E_{s}=\int_{\Gamma_{C}}\left|u_{-}^{\delta}-\tilde{u}_{-}^{\delta, k}\right|^{2} d s
$$

and the relative error function

$$
E_{r}=\frac{\int_{\Gamma_{C}}\left|u_{-}^{\delta}-\tilde{u}_{-}^{\delta, k}\right|^{2} d s}{\int_{\Gamma_{C}}\left|u_{-}^{\delta}\right|^{2} d s}
$$

where $\Gamma_{C}$ is the measurement circle with radius $C$. In the following, various exact and approximate results are given.

As a first example, to verify the accuracy of the exact solution for the Dirichlet case, FEM and analytic solutions are compared. In the application of the FEM solution, the discretization size is roughly $\lambda / 42$, and the radius of the approximate boundary condition (ABC) given in (63) is $2.5 \lambda$ where $\lambda$ is the free space wavelength. We consider as a coating a cylinder having a radius $5 \lambda / 6$ coated over a perfect electric conductor (PEC) which is a cylinder of radius $0.75 \lambda$. The coating with a thickness of $\lambda / 12$ is made of a material with conductivity of $0.2\left(S . \mathrm{m}^{-1}\right)$ and relative dielectric permittivity of $3 \epsilon_{0}$ where $\epsilon_{0}$ is dielectric permittivity of free space. The cylinder is illuminated by a time harmonic electromagnetic wave with frequency $5 \mathrm{MHz}$ and incidence direction is 0 . In other words, the wavelength of incident wave is selected as $0.6 \mathrm{~m}$. The amplitude and phase of the total fields which are shown in Figure 2 are obtained on the circle $\Gamma_{C}$ with radius $5 \lambda / 3$. 

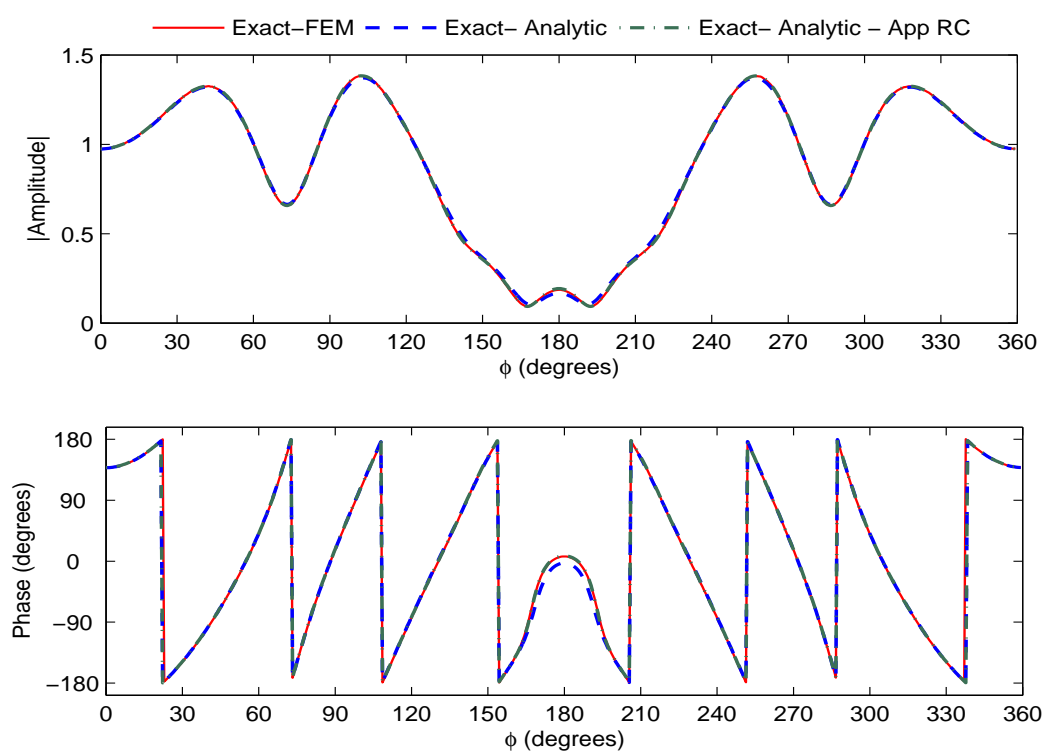

Figure 2: Ex 1. Comparisons of the amplitude and the complex phase of the total field on a circle with radius $\Gamma_{C}=5 \lambda / 3$ obtained by exact solutions for the circular coating with thickness of $\delta=\lambda / 12$ in the Dirichlet case

Obviously the results of FEM and analytic solutions are in a good agreement. The error and relative error given (87) and (88) are $E_{s}=2.40861 e^{-3}$ and $E_{r}=$ $0.15670 e^{-3}$, respectively. Taking into account the $\mathrm{ABC}$ in analytic solution, smaller errors are calculated as $E_{s}=0.58408 e^{-3}$ and $E_{r}=0.10627 e^{-3}$. In the following examples we omit the small difference caused by ABC.

As a second example, a PEC cylinder of radius $0.46 \lambda$ coated by a cylinder material of radius $0.5 \lambda$ with relative dielectric permittivity of $3.5 \epsilon_{0}$ and conductivity of $0.3\left(S . \mathrm{m}^{-1}\right)$ taken into account. The object is illuminated at 6 $\mathrm{MHz}$ with incidence angle $\pi$. The discretization size is roughly $\lambda / 55$, and the radius of the $\mathrm{ABC}$ is $2 \lambda$. The amplitude and the phase of the total field on the circle $\Gamma_{C}$ with radius $1.6 \lambda$ obtained through FEM end analytic solution are compared in Figure 3. The error and relative error are $E_{s}=7.75322 e^{-3}$ and $E_{r}=0.09767 e^{-3}$, respectively.

Similarly, to verify the accuracy of the exact solution for the Neumann case, the FEM and analytic solutions are compared. As a third example, a perfect magnetic conductor (PMC) cylinder of radius $5 \lambda / 6$ coated by a cylinder material of radius $0.75 \lambda$ with relative dielectric permittivity of $2.5 \epsilon_{0}$ and conductivity of $0.2\left({\left.\mathrm{~S} . \mathrm{m}^{-1}\right)}\right.$ is considered. The object is illuminated at $5 \mathrm{MHz}$ with incidence angle $\pi / 4$. The discretization size is roughly $\lambda / 44$, and the radius of the $\mathrm{ABC}$ is $2.5 \lambda$. The amplitude and the phase of the total field on the circle $\Gamma_{C}$ with radius $5 \lambda / 3$ obtained through FEM end analytic solution are compared in Figure 4. The error and relative error are $E_{s}=2.59305 e^{-3}$ and $E_{r}=0.16496 e^{-3}$, respectively.

As a next example, a PMC cylinder of radius $0.97 \lambda$ coated by a cylinder material of radius $\lambda$ with relative dielectric permittivity of $3 \epsilon_{0}$ and conductivity 

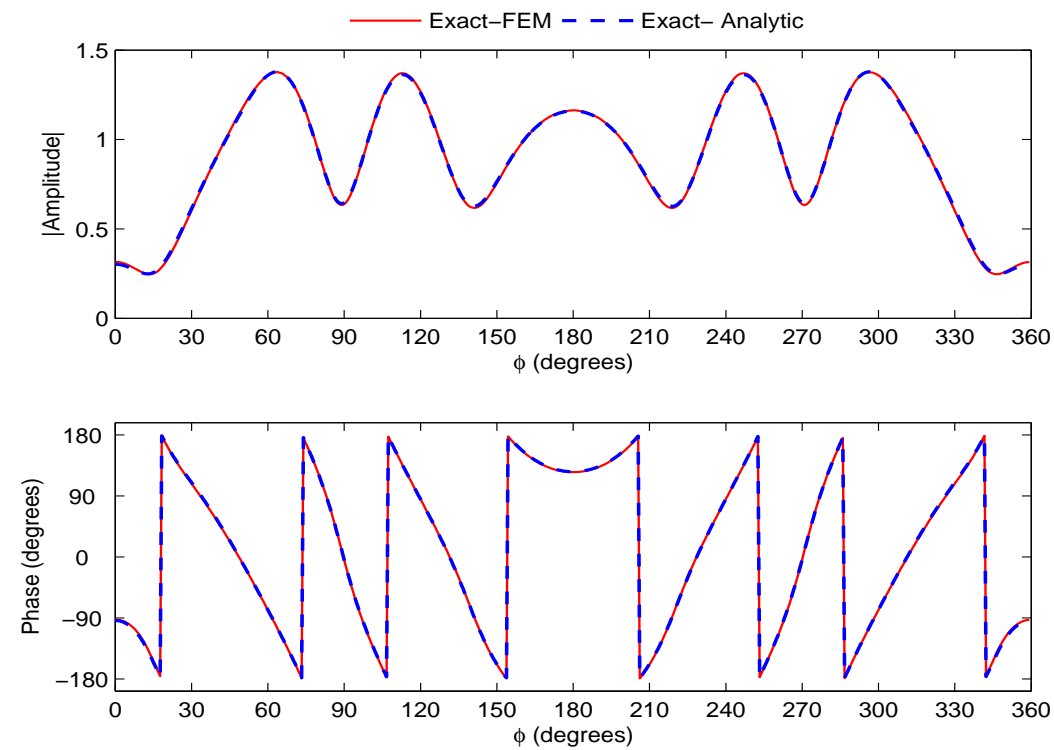

Figure 3: Ex 2. Comparisons of the amplitude and the complex phase of the total field on a circle with radius $\Gamma_{C}=1.6 \lambda$ obtained by exact solutions for the circular coating with thickness of $\delta=\lambda / 25$ in the Dirichlet case
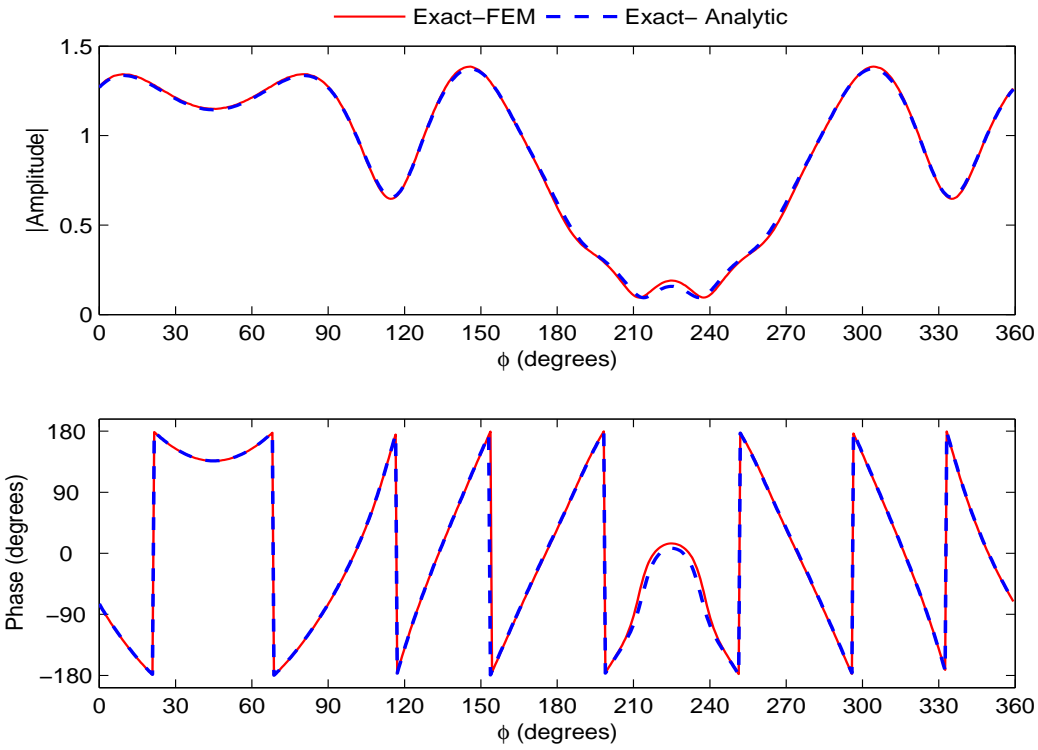

Figure 4: Ex 3. Comparisons of the amplitude and the complex phase of the total field on a circle with radius $\Gamma_{C}=5 \lambda / 3$ obtained by exact solutions for the circular coating with thickness of $\delta=\lambda / 12$ in the Neumann case 

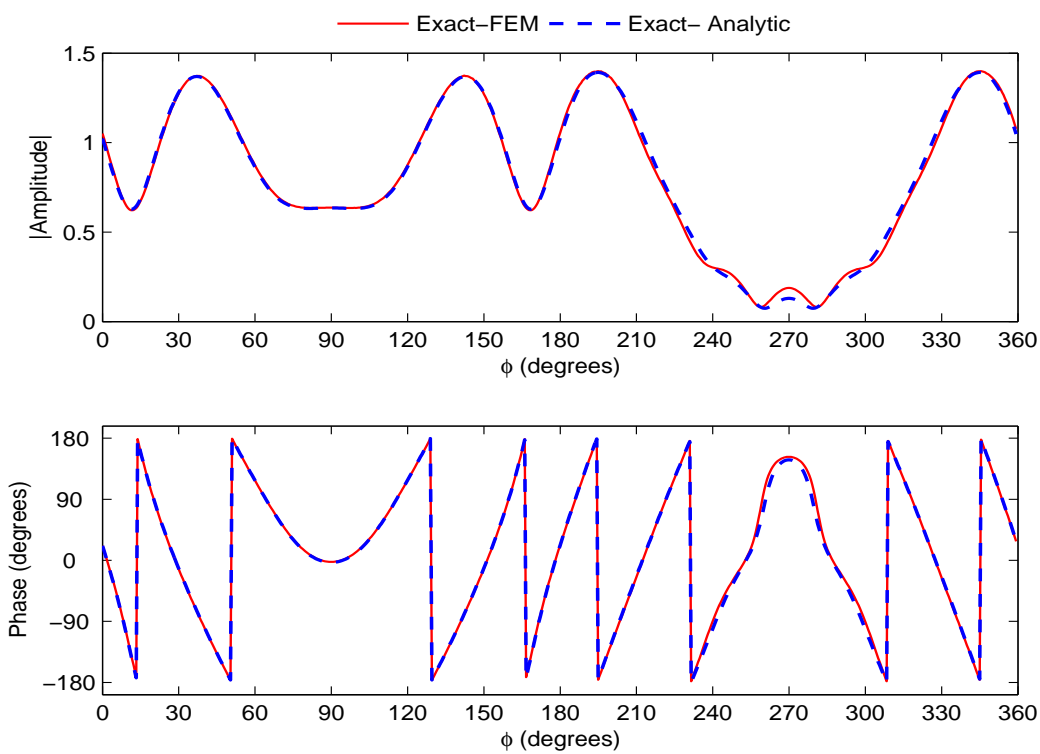

Figure 5: Ex 4. Comparisons of the amplitude and the complex phase of the total field on a circle with radius $\Gamma_{C}=2 \lambda$ obtained by exact solutions for the circular coating with thickness of $\delta=0.03 \lambda$ in the Neumann case

of $0.4\left(S . \mathrm{m}^{-1}\right)$ taken into account. The thinner coated object is illuminated at 3 $\mathrm{MHz}$ with incidence angle $\pi / 2$. The discretization size is roughly $\lambda / 48$, and the radius of the $\mathrm{ABC}$ is $2.2 \lambda$. The amplitude and the phase of the total field on the circle $\Gamma_{C}$ with radius $2 \lambda$ obtained through FEM end analytic solution are compared in Figure [5] The error and relative error are $E_{s}=11.58471 e^{-3}$ and $E_{r}=0.45152 e^{-003}$, respectively.

The above examples show that FEM is sufficient converge. In the following, various exact and approximate results are given for the Dirichlet case and Neumann,respectively.

\subsection{Numerical Results for the Dirichlet case}

In this section, the numerical results of the problem is given to verify the accuracy and effectiveness of the GIBC's for the Dirichlet case. If one substitutes the error function Es given in (87) into the condition given in (13) and logarithm to both sides

$$
\log (E s) \simeq \log (c)+2(m+1) \log \left(\delta_{0}\right)
$$

is obtained where $c$ is a constant. [89] is an equation of a line whose slope is $2(\mathrm{~m}+1)$. The exact solution and approximate solutions for order 1, 2, 3 are compared for various examples.

As a first example, a PEC cylinder coated by a cylinder material of radius $5 \lambda / 6$ with relative dielectric permittivity of $3.0 \epsilon_{0}$ and conductivity of $0.1\left(S . m^{-1}\right)$ is considered. $f(s)$ is selected as 1 . Note that, $\delta(s)=\delta_{0} f(s)$ was given in (23). The object is illuminated at $5 \mathrm{MHz}$ with incidence angle $\pi / 3$. The discretization 


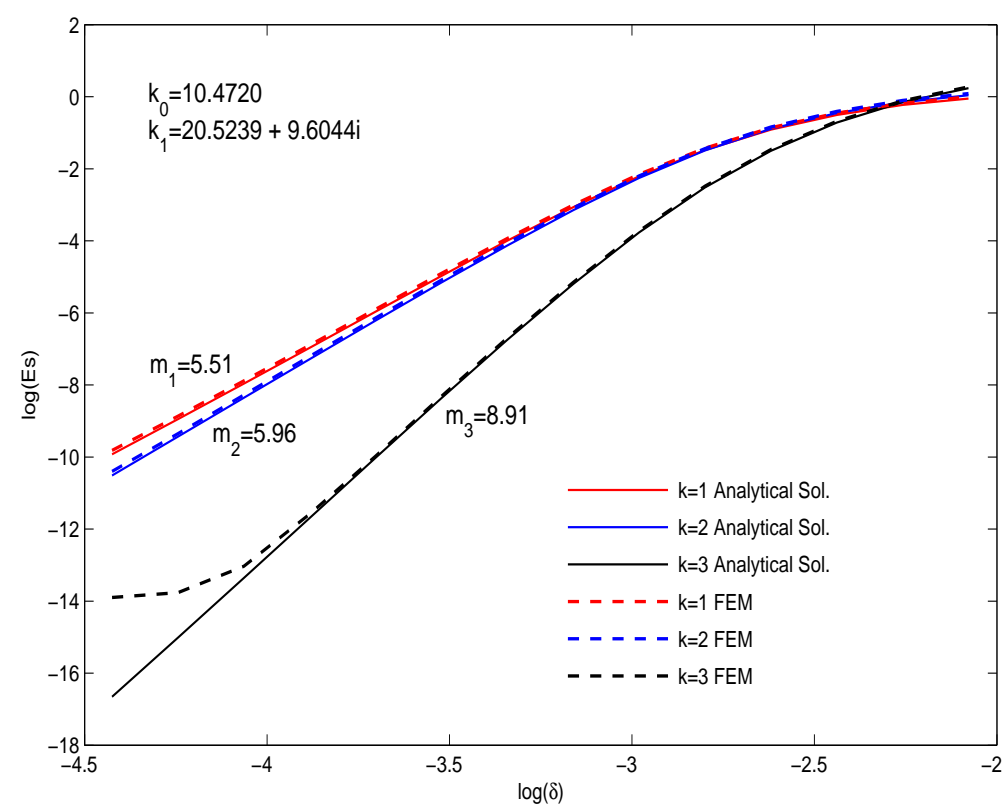

Figure 6: Ex 1. The graphics of $\log (E s)$ given in (89) for the circular coating with radius $5 \lambda / 6$ and constant thickness in the Dirichlet case

size is roughly $\lambda / 58$, and the radius of the $\mathrm{ABC}$ is $2.5 \lambda$. The errors of total field Es's are calculated on the circle $\Gamma_{C}$ with radius $5 \lambda / 3$ and for order 1,2 , and 3 the equation of the lines given in (89) are compared in Figure 6] Furthermore, the lines are derived for analytical solutions to verify the accuracy of FEM. The slope of lines for order 1,2 and 3 are approximately $m_{1}=5.51, m_{2}=5.96$ and $m_{3}=8.91$, respectively.

In the first example curvature was constant. As a second example, an elliptical cross-sectioned dielectric coating with relative dielectric permittivity of $4.0 \epsilon_{0}$ and conductivity of $0.05\left(S . \mathrm{m}^{-1}\right)$ is taken into account. The curvature, is not constant, is given by

$$
c(t)=\frac{-a b}{\left(a^{2} \sin ^{2}(t)+b^{2} \cos ^{2}(t)\right)^{3 / 2}}
$$

where $a=3 \lambda / 5$ and $b=2 \lambda / 5$ are parameters in parametric equations of the elliptical surface given by

$$
\left\{\begin{array}{l}
x(t)=a \cos (t) \\
y(t)=b \sin (t)
\end{array}, a>0, b>0\right.
$$

$f(s)$ is selected as 1 . In other words, the width $\delta(s)$ is constant. The object is illuminated at $6 \mathrm{MHz}$ with incidence angle $\pi / 4$. The discretization size is roughly $\lambda / 75$, and the radius of the ABC is $2 \lambda$. The errors of total field Es's are calculated on the circle $\Gamma_{C}$ with radius $8 \lambda / 5$ and for order 1,2 , and 3 equation 


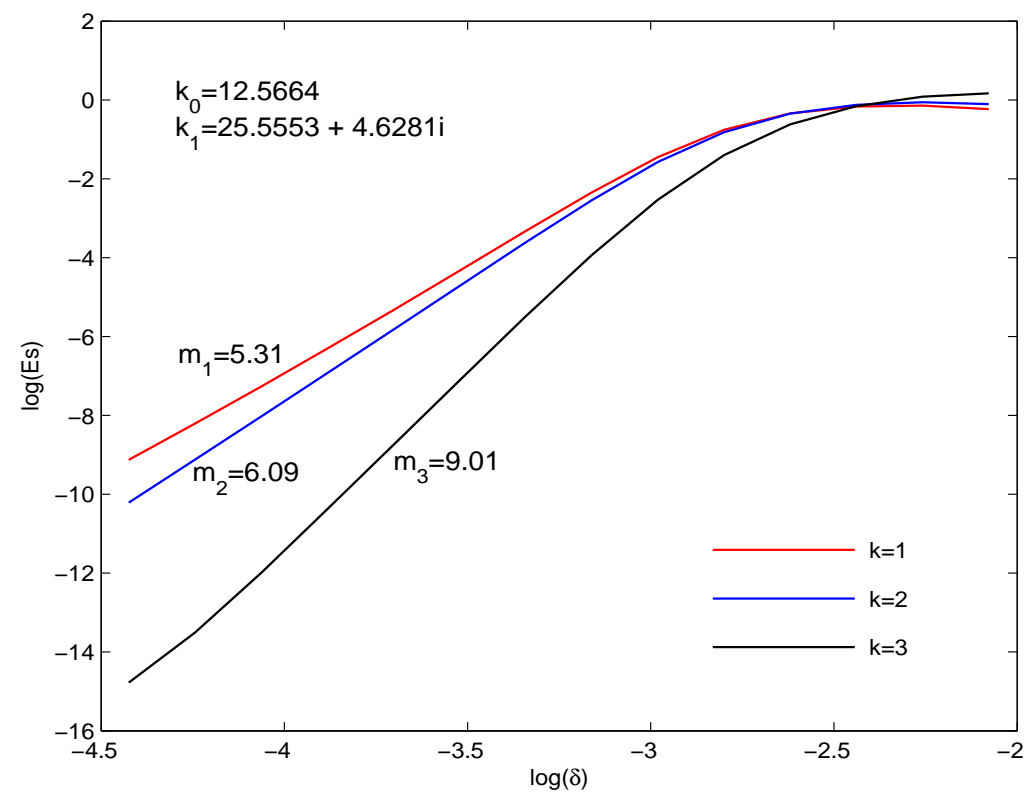

Figure 7: Ex 2. The graphics of $\log (E s)$ given in (89) for the object with constant thickness given by (90) where $a=3 \lambda / 5$ and $b=2 \lambda / 5$ in the Dirichlet case 
of lines given in (89) are compared in Figure 7 The slope of lines for order 1,2 and 3 are approximately $m_{1}=5.31, m_{2}=6.09$ and $m_{3}=9.01$, respectively.

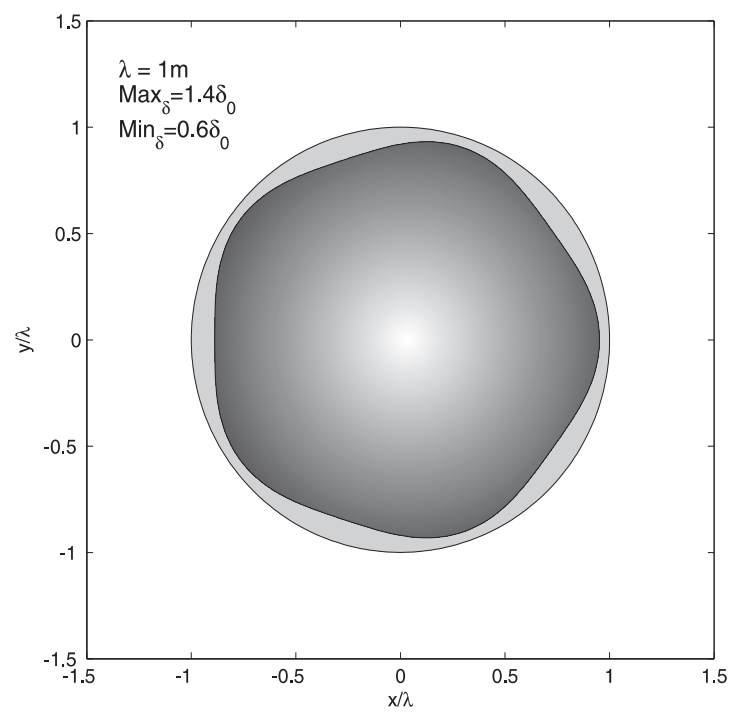

Figure 8: Ex 3. The geometry of the circular coating with radius $\lambda$ whose width is given in (91)

As a third example, a cylindrical cross-sectioned dielectric coating of variable width, with relative dielectric permittivity of $3.0 \epsilon_{0}$ and conductivity of $0.3\left(S . \mathrm{m}^{-1}\right)$ is taken into account. The radius of coating is selected as $\lambda$, which is relatively bigger(see Figure 8). The variable of width is given with equation

$$
\delta(t)=\delta_{0}(1-0.4 \sin (5 t))
$$

Considering the equation (91), It can be seen that $f(t)=(1-0.4 \sin (5 t))$. The object is illuminated at $3 \mathrm{MHz}$ with incidence angle $\pi / 2$. The discretization size is roughly $\lambda / 55$, and the radius of the $\mathrm{ABC}$ is $2.2 \lambda$. The errors of total field Es's are calculated on the circle $\Gamma_{C}$ with radius $2 \lambda$ and for order 1,2 , and 3 , the equation of the lines are compared in Figure 9] The slope of lines for order 1,2 and 3 are approximately $m_{1}=5.68, m_{2}=5.75$ and $m_{3}=9.19$, respectively.

As a fourth example, an elliptical cross-sectioned dielectric coating of variable width, with relative dielectric permittivity of $2.0 \epsilon_{0}$ and conductivity of $0.1\left(S . m^{-1}\right)$ is taken into account. The boundary of the coating is given in (90) where $a=1.5 \lambda$ and $b=1.2 \lambda$. The variable of width is given with equation

$$
\delta(t)=\delta_{0}(1-0.4 \sin (6 t))
$$

The object (see Figure 10) is illuminated at $3 \mathrm{MHz}$ with incidence angle $\pi / 4$. The discretization size is roughly $\lambda / 50$, and the radius of the $\mathrm{ABC}$ is $2.8 \lambda$. The errors of total field Es's are calculated on the circle $\Gamma_{C}$ with radius $2.5 \lambda$ and for order 1, 2, and 3, the equation of the lines are compared in Figure [1] The coating is large, curvature of its boundary is variable, and its width is variable, 


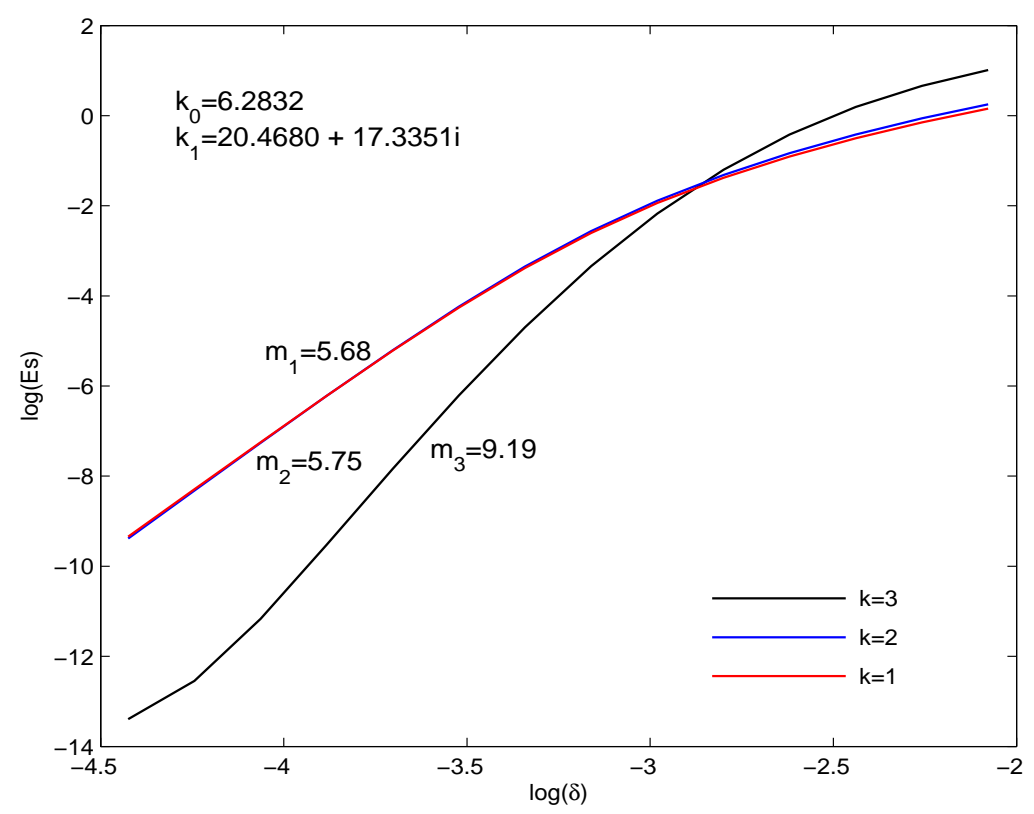

Figure 9: Ex 3. The graphics of $\log (E s)$ given in (89) for the object illustrated in Figure 8 in the Dirichlet case

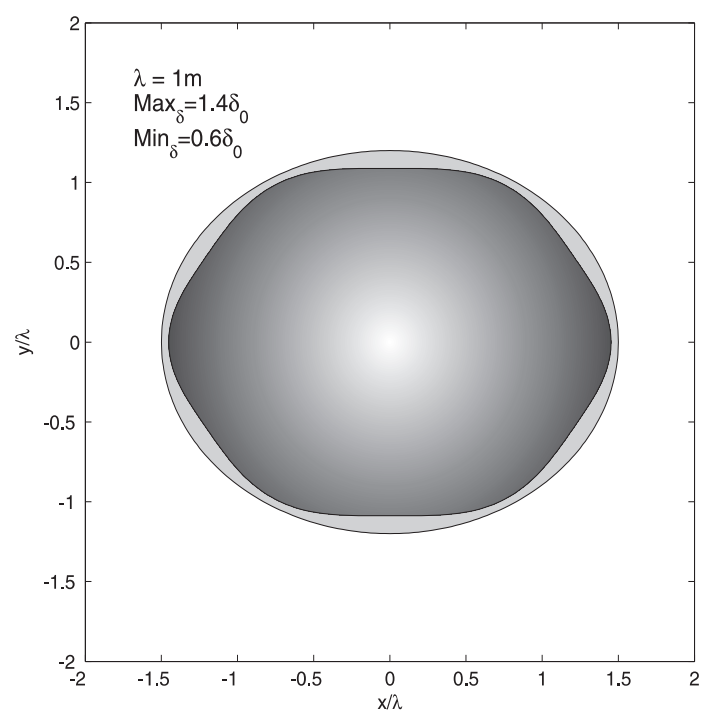

Figure 10: Ex 4. The geometry of the elliptical coating whose boundary is given in (90) where $a=1.5 \lambda, b=1.2 \lambda$ and width is given in (92) 


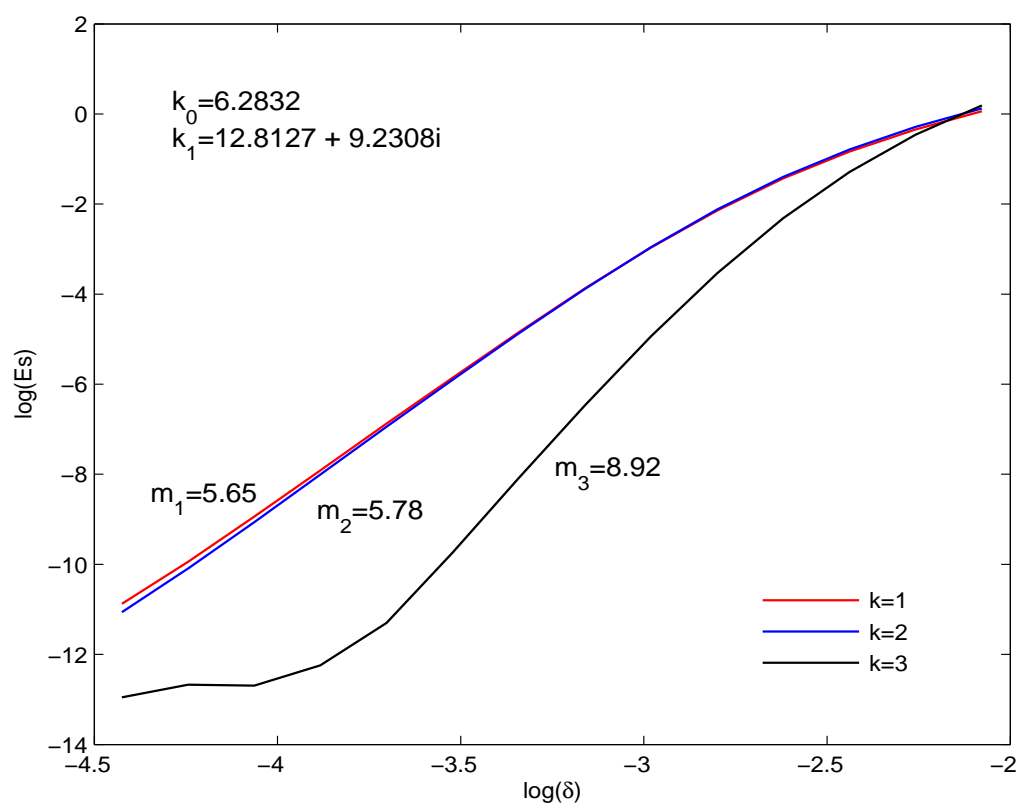

Figure 11: Ex 4. The graphics of $\log (E s)$ given in (89) for the object illustrated in Figure 10] in the Dirichlet case

but the expected results are obtained as results of previous examples. The slope of lines for order 1,2 and 3 are approximately $m_{1}=5.65, m_{2}=5.78$ and $m_{3}=8.92$, respectively.

In the next example, a more complex coating whose boundary is given by parametric equation

$$
X_{\Gamma}=\left(\begin{array}{c}
(A+B \sin (6 t)+C \cos (5 t)+D \cos (6 t)) \cos (t) \\
(A+B \sin (6 t)+C \cos (5 t)+D \cos (6 t)) \sin (t)
\end{array}\right), t \in[0,2 \pi)
$$

where $A=\lambda, B=0.06 \lambda, C=0.07 \lambda$ and $D=0.05 \lambda$ and the width is given by equation

$$
\delta(t)=\delta_{0}(1-0.4 \sin (7 t))
$$

is taken into account(see Figure 12). Thus, the parametric equation of the PEC can be shown as

$$
x_{\Gamma^{\delta}}(t)=x_{\Gamma}(t)+\delta(t) \vec{n}(t), t \in[0,2 \pi)
$$

The coating with relative dielectric permittivity of $3.0 \epsilon_{0}$ and conductivity of $0.05\left(S . m^{-1}\right)$ is illuminated at $3 \mathrm{MHz}$ with incidence angle $\pi / 3$. The discretization size is roughly $\lambda / 55$, and the radius of the $\mathrm{ABC}$ is $2.2 \lambda$. The errors of total field Es's are calculated on the circle $\Gamma_{C}$ with radius $2 \lambda$ and for order 1,2 , and 3 , the equation of the lines are compared in Figure 13 The slope of lines for order 1,2 and 3 are approximately $m_{1}=5.26, m_{2}=5.98$ and $m_{3}=9.23$, respectively. 


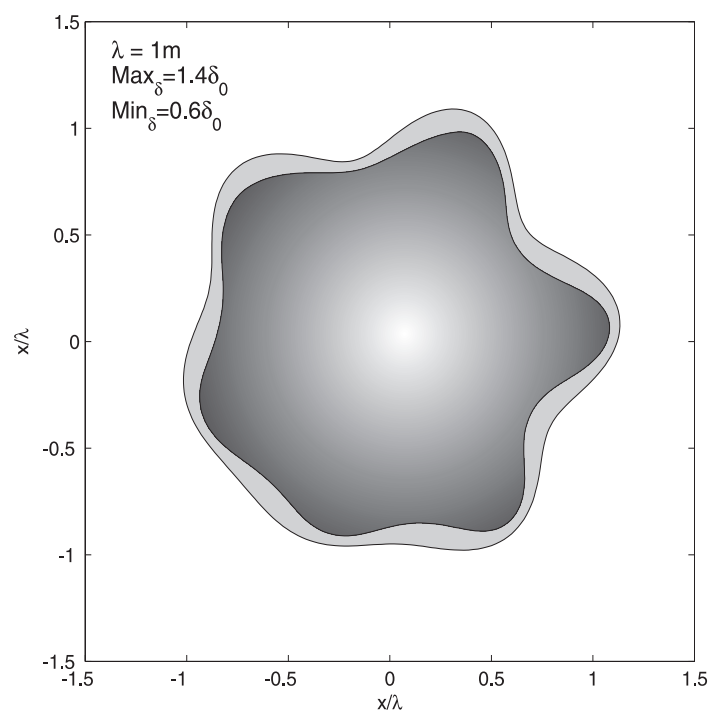

Figure 12: Ex 5. The geometry of the object whose boundary is given in (93) where $A=\lambda, B=0.06 \lambda, C=0.07 \lambda, D=0.05 \lambda$ and width is given in (94)



Figure 13: Ex 5. The graphics of $\log (E s)$ given in (89) for the object illustrated in Figure 12 in the Dirichlet case 


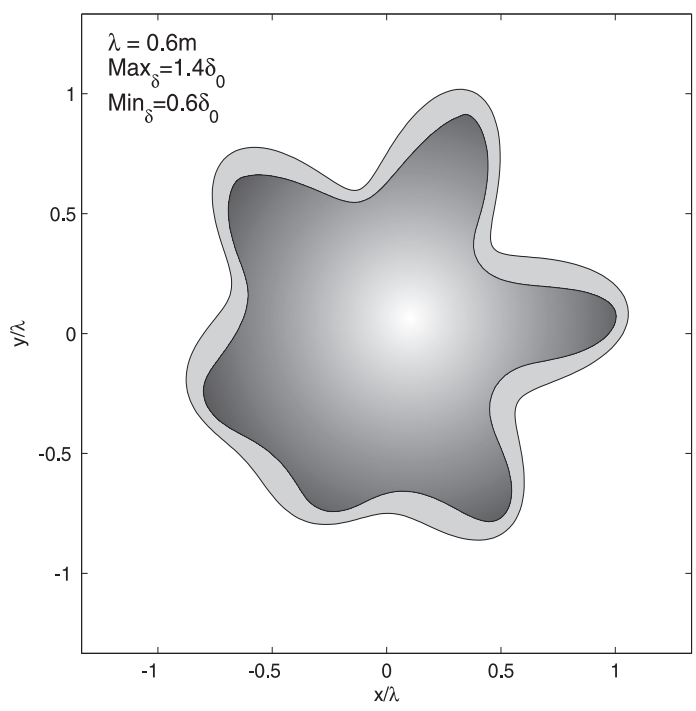

Figure 14: Ex 6. The geometry of the object whose boundary is given in (93) where $A=5 \lambda / 6, B=0.1 \lambda, C=7 \lambda / 60, D=\lambda / 12$ and width is given in (94) where $\delta_{0}=\lambda / 12$

As a next example, similar shaped coating whose boundary is given in (93) where $A=5 \lambda / 6, B=0.1 \lambda, C=7 \lambda / 60$ and $D=\lambda / 12$ and width is given in (94) where $\delta_{0}=\lambda / 12$ is taken into account(see Figure 14). The coating with relative dielectric permittivity of $2.5 \epsilon_{0}$ and conductivity of $0.01\left(S . \mathrm{m}^{-1}\right)$ is illuminated at $5 \mathrm{MHz}$ with incidence angle $\pi / 3$. The radius of the $\mathrm{ABC}$ is 2.5 $\lambda$. The amplitude and the phase of the total field on the circle $\Gamma_{C}$ with radius $5 \lambda / 3$ obtained through exact end approximate solutions are compared in Figure 15. The errors are $E_{s}=52.24118^{-3}, E_{s}=38.33849 e^{-3}, E_{s}=1.84402 e^{-3}$ and relative errors are $E_{r}=0.56314 e^{-3}, E_{r}=0.45986 e^{-3}, E_{r}=0.09751 e^{-3}$, respectively.

As a next example, a kite shaped dielectric coating, with relative dielectric permittivity of $2.5 \epsilon_{0}$ and conductivity of $0.01\left(\mathrm{~S}_{\mathrm{m}} \mathrm{m}^{-1}\right)$, whose boundary is given by parametric equation

$$
X_{\Gamma}=\left(\begin{array}{l}
A \cos (t)+B \cos (2 t)-C \\
A \sin (t)
\end{array}\right), t \in[0,2 \pi)
$$

where $A=\lambda, B=11 \lambda / 30$ and $C=\lambda / 60$ is taken into account(see Figure [16). The boundary of PEC is given in equation (95) where

$$
\delta(t)=\delta_{0}(1-0.4 \sin (6 t))
$$

The object is illuminated at $5 \mathrm{MHz}$ with incidence angle $\pi / 3$. The discretization size is roughly $\lambda / 50$, and the radius of the $\mathrm{ABC}$ is $8 \lambda / 3$. The errors of total field Es's are calculated on the circle $\Gamma_{C}$ with radius $2 \lambda$ and for order 1,2 , and 3 , the equation of the lines are compared in Figure 17 The slope of lines for order 1,2 and 3 are approximately $m_{1}=5.31, m_{2}=5.91$ and $m_{3}=9.33$, respectively. 

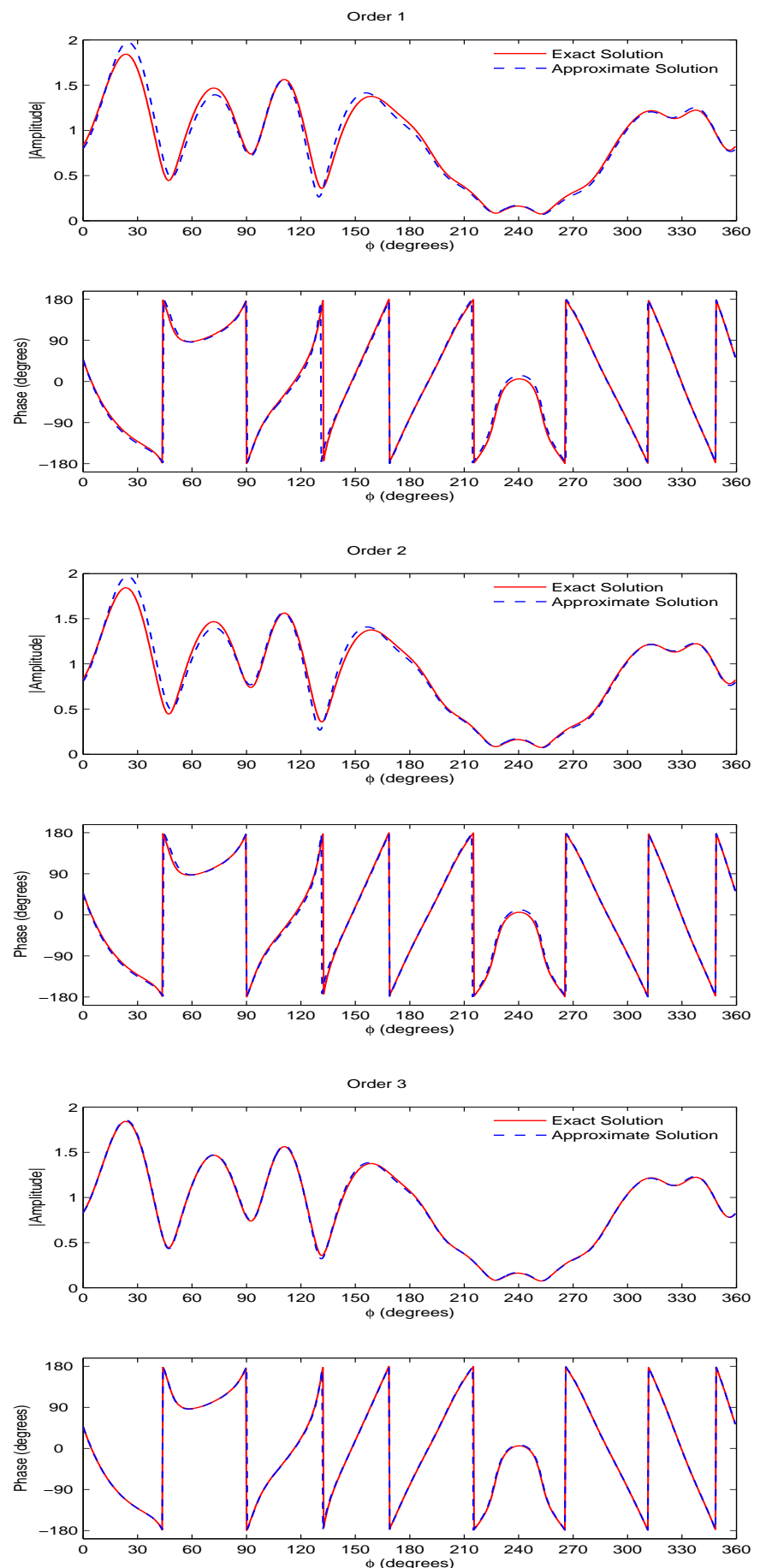

Figure 15: Ex 6. Comparisons of the amplitude and the complex phase of the total field on a circle with radius $\Gamma_{C}=5 \lambda / 3$ obtained by FEM and approximate solutions for the object illustrated in Figure 14 in the Dirichlet case 


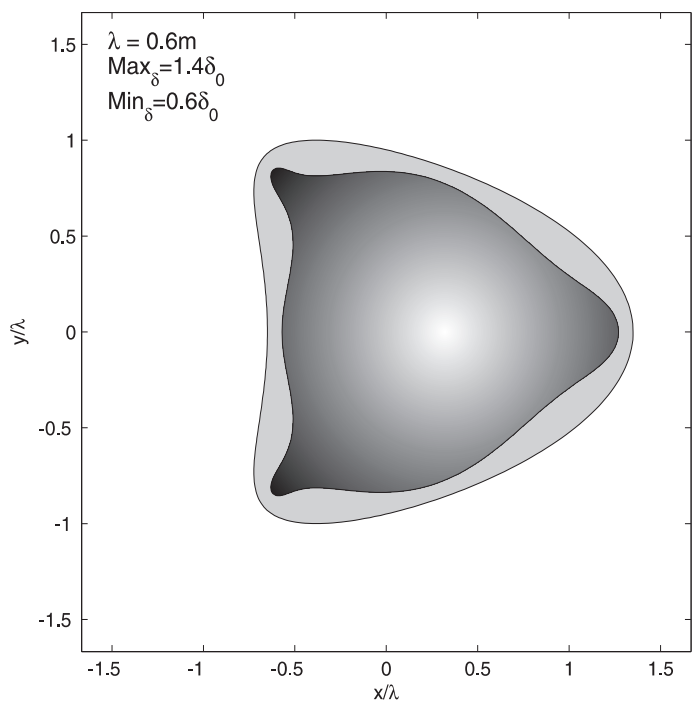

Figure 16: Ex 7. The geometry of the object whose boundary is given in (96) where $A=\lambda, B=11 \lambda / 30, C=\lambda / 60$ and width is given in (97)

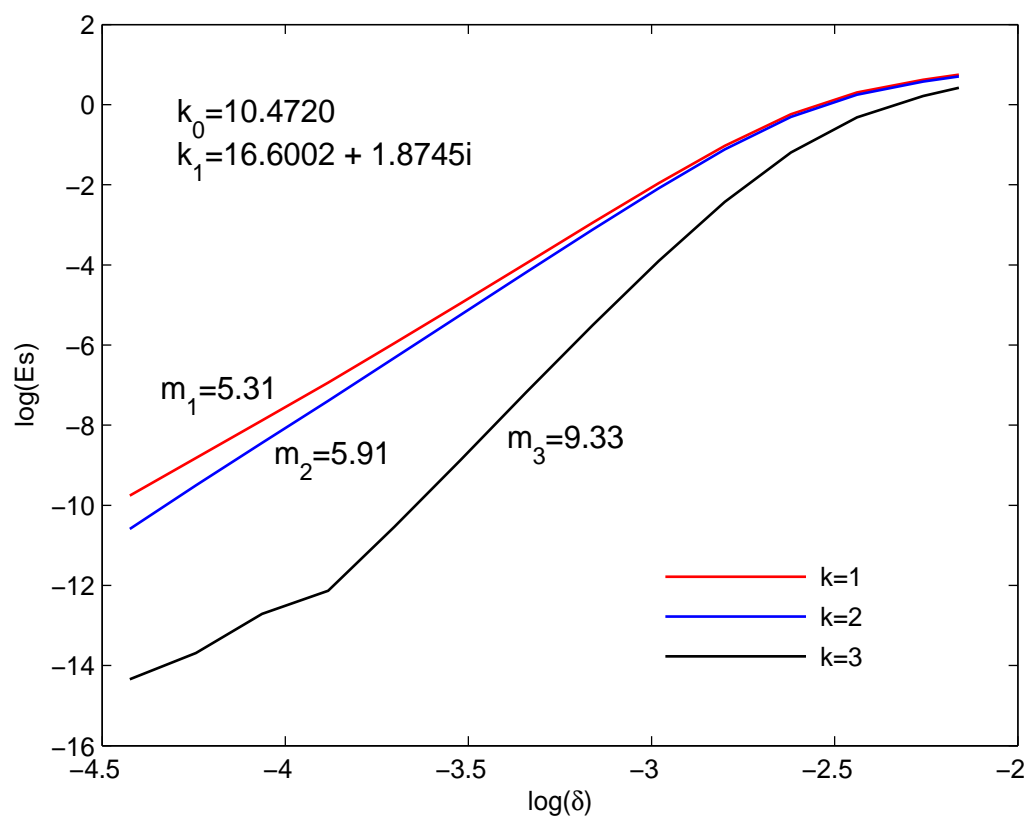

Figure 17: Ex 7. The graphics of $\log (E s)$ given in (89) for the object illustrated in Figure 16 in the Dirichlet case 


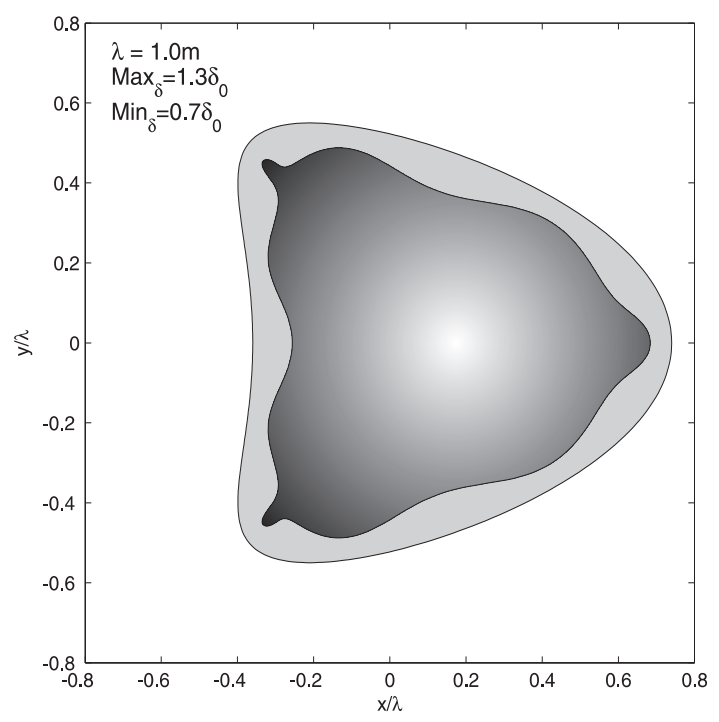

Figure 18: Ex 8. The geometry of the object whose boundary is given in (96) where $A=0.55 \lambda, B=0.2 \lambda, C=0.01 \lambda$ and width is given in (98)

As a next example, similar shaped coating whose boundary is given in (96) where $A=0.55 \lambda, B=0.2 \lambda, C=0.01 \lambda$ and width is given as

$$
\delta(t)=0.08 \lambda(1-0.3 \sin (9 t))
$$

is taken into account(see Figure 18). The coating with relative dielectric per-

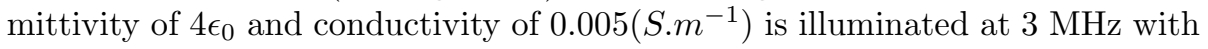
incidence angle $\pi / 3$. The radius of the ABC is $1.5 \lambda$. The amplitude and the phase of the total field on the circle $\Gamma_{C}$ with radius $1.2 \lambda$ obtained through exact end approximate solutions are compared in Figure 19] The errors are $E_{s}=180.80255^{-3}, E_{s}=146.41781^{-3}, E_{s}=25.56597 e^{-3}$ and relative errors are $E_{r}=1.16502 e^{-3}, E_{r}=1.02404 e^{-3}, E_{r}=0.41652 e^{-3}$, respectively.

In all previous examples $\delta(s)$ was assumed to be regular. As next examples in this chapter, we assume that $\delta(s)$ is a non-regular function. To put it more clearly, $\delta^{\prime}(s)$ is not continuous and $\delta^{\prime \prime}(s)$ is not defined at every point. Redefining the expressions over $\Gamma$ as piecewise continuous functions and vanishing the expressions $\delta^{\prime \prime}(s)$, the variational formulations for order 1, 2 and 3 given in 69 [71 73 and 75] can be rearranged. We assume that $\delta^{\prime}(s)$ or curvature $c(s)$ is not continuous at $\mathrm{N}$ points. Selecting the $\mathrm{N}$ points as limits of integrals, one can modify the variational formulation for order 1 (69) as

$$
\begin{array}{r}
\int_{\Omega_{-}} \nabla u_{-}^{\delta} \nabla \bar{\nu}-k_{0}^{2} \int_{\Omega_{-}} u_{-}^{\delta} \bar{\nu}+\sum_{n=1}^{N} \int_{\Gamma_{n}} \frac{1}{\delta(s)} u_{-}^{\delta} \bar{\nu} d s \\
-\int_{\Gamma_{R}} i k_{0} u_{-}^{\delta} \bar{\nu} d s-\int_{\Gamma_{R}}\left(\frac{\partial u^{i}}{\partial \bar{n}}-i k_{0} u^{i}\right) \bar{\nu} d s=0
\end{array}
$$



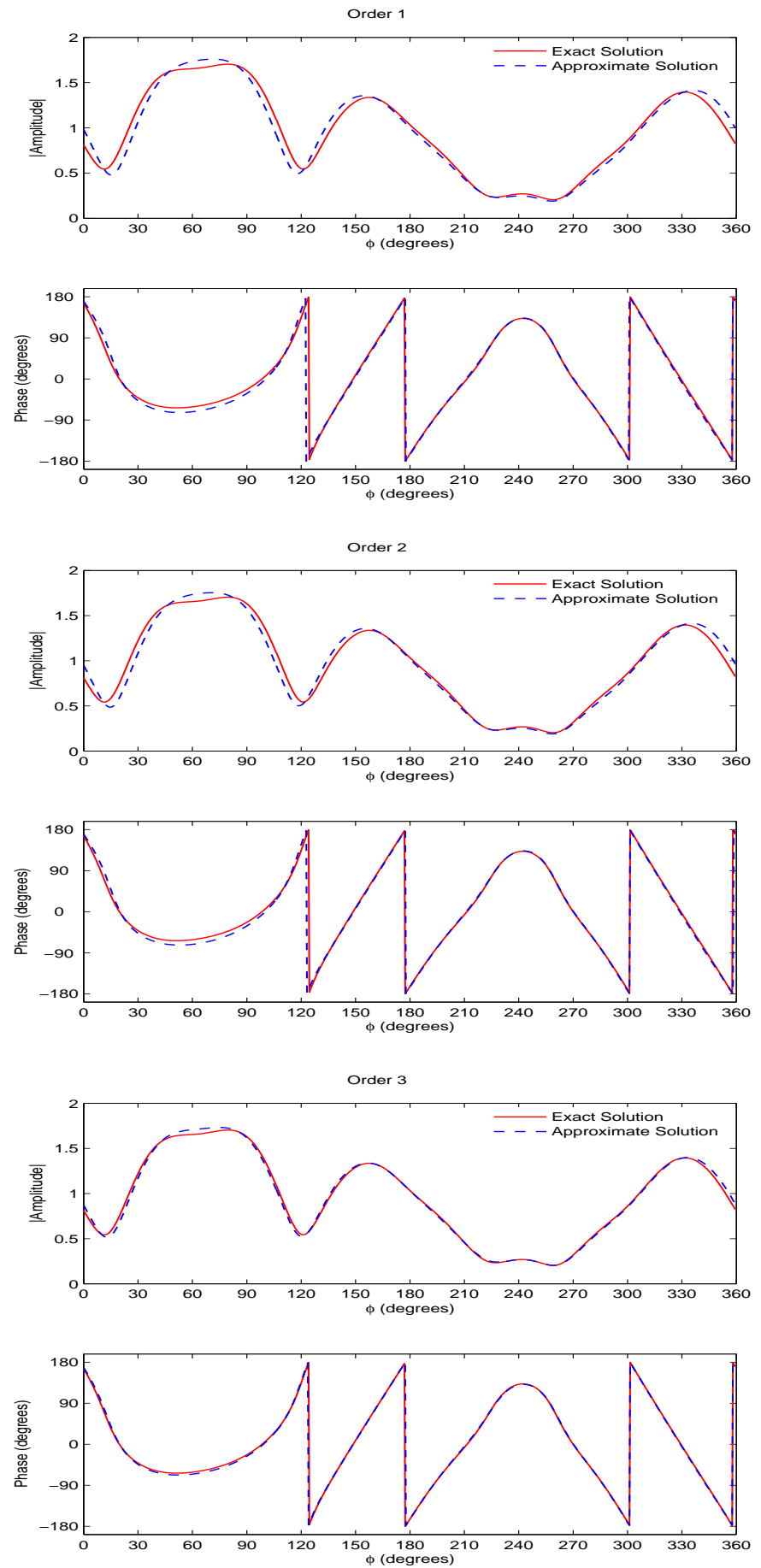

Figure 19: Ex 8. Comparisons of the amplitude and the complex phase of the total field on a circle with radius $\Gamma_{C}=1.2 \lambda$ obtained by FEM and approximate solutions for the object illustrated in Figure 18 in the Dirichlet case 
where $\cup_{1}^{N} \Gamma_{n}=\Gamma$. Similarly, for order 2

$$
\begin{array}{r}
\int_{\Omega_{-}} \nabla u_{-}^{\delta} \nabla \bar{\nu}-k_{0}^{2} \int_{\Omega_{-}} \bar{\nu} u_{-}^{\delta}+\sum_{n=1}^{N} \int_{\Gamma_{n}} \frac{1}{\delta(s)\left(1-\frac{1}{2} \delta(s) c(s)\right)} u_{-}^{\delta} \bar{\nu} d s \\
-\int_{\Gamma_{R}} i k_{0} u_{-}^{\delta} \bar{\nu} s-\int_{\Gamma_{R}}\left(\frac{\partial u^{i}}{\partial \bar{n}}-i k_{0} u^{i}\right) \bar{\nu} s=0
\end{array}
$$

is obtained. By partial integration of the $\int_{\Gamma} \frac{1}{2} \delta^{2}(s) \delta^{\prime \prime}(s) \psi \bar{\nu}_{2} d s$, the variational formulation for order 3 (75) can be denotes as

$$
\begin{array}{r}
\sum_{n=1}^{N} \int_{\Gamma_{n}} u_{-}^{\delta} \bar{\nu}_{2} d s+\sum_{n=1}^{N} \int_{\Gamma_{n}} \alpha \psi \bar{\nu}_{2} d s+\sum_{n=1}^{N} \int_{\Gamma_{n}} \beta \frac{\partial \psi}{\partial s} \bar{\nu}_{2} d s \\
+\sum_{n=1}^{N} \int_{\Gamma_{n}} \beta \psi \frac{\partial \bar{\nu}_{2}}{\partial s} d s+\sum_{n=1}^{N} \int_{\Gamma_{n}} \gamma \frac{\partial \psi}{\partial s} \frac{\partial \bar{\nu}_{2}}{\partial s} d s=0
\end{array}
$$

where

$$
\begin{gathered}
\alpha=\delta(s)\left(1-\frac{1}{2} \delta(s) c(s)+\frac{1}{3} \delta^{2}(s) c^{2}(s)\right)+\frac{1}{3} \delta^{3}(s) k_{1}^{2}-\delta(s)\left(\delta^{\prime}(s)\right)^{2} \\
\beta=-\frac{1}{2} \delta^{2}(s) \delta^{\prime}(s) \\
\gamma=-\frac{1}{3} \delta^{3}(s)
\end{gathered}
$$

Also, the other variational formulation (173) $)$ can be modify as

$$
\begin{array}{r}
\int_{\Omega_{-}} \nabla u_{-}^{\delta} \nabla \bar{\nu}-k_{0}^{2} \int_{\Omega_{-}} u_{-}^{\delta} \bar{\nu}-\sum_{n=1}^{N} \int_{\Gamma_{n}} \psi \bar{\nu} d s-\int_{\Gamma_{R}} i k_{0} u_{-}^{\delta} \bar{\nu} d s \\
-\int_{\Gamma_{R}}\left(\frac{\partial u^{i}}{\partial \bar{n}}-i k_{0} u^{i}\right) \bar{\nu} d s=0
\end{array}
$$

As a last example, dielectric coating of non-regular width is displayed in Figure 20 is taken into account. The maximum width and length of the object are $23 \lambda / 6$ and $13 \lambda / 6$, respectively. The object is illuminated at $5 \mathrm{MHz}$ with incidence angle $\pi / 3$. The discretization size is roughly $\lambda / 44$, and the radius of the ABC is $10 \lambda / 3$. The errors of total field Es's are calculated on the circle $\Gamma_{C}$ with radius $3 \lambda$. When dielectric coating's relative dielectric permittivity and conductivity are selected as respectively $3 \epsilon_{0}$ and $0.1\left(S . m^{-1}\right)$, order 1,2 , and 3 solutions are compared in Figure 21. The slope of lines for order 1,2 and 3 are approximately $m_{1}=5.45, m_{2}=5.80$ and $m_{3}=9.14$, respectively. Selecting $\delta_{0}$ as $\lambda / 12$, the amplitude and the phase of the total field are compared in Figure 22 The errors are $E_{s}=233.22764^{-3}, E_{s}=224.30272^{-3}, E_{s}=57.87359 e^{-3}$ and relative errors are $E_{r}=1.45933 e^{-3}, E_{r}=1.43530 e^{-3}, E_{r}=0.70211 e^{-3}$, respectively. Selecting relative dielectric permittivity and conductivity as respectively $5 \epsilon_{0}$ and $0.05\left(S . \mathrm{m}^{-1}\right)$, results displayed in Figure 23 is obtained. The slope of lines are $m_{1}=5.69, m_{2}=6.08$ and $m_{3}=9.68$. 


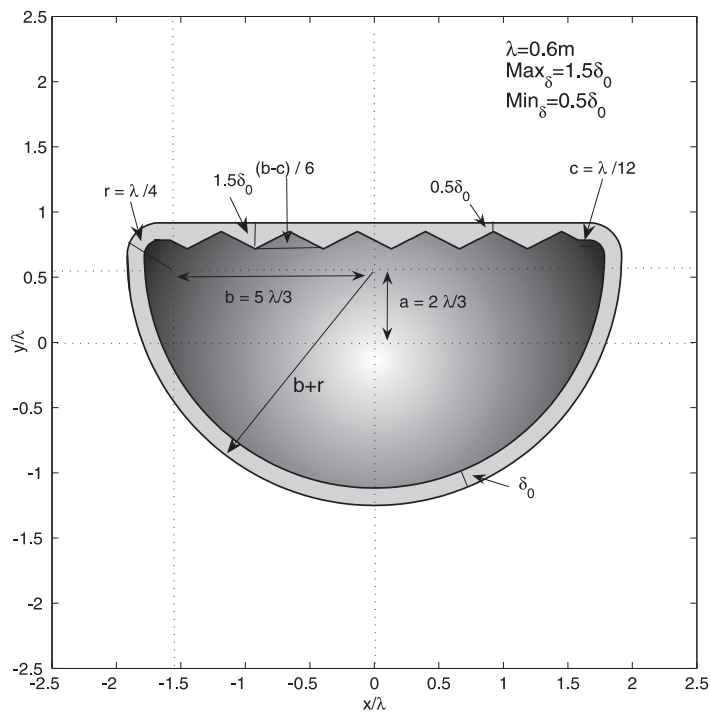

Figure 20: Ex 9. The geometry of the coating with non-regular thickness

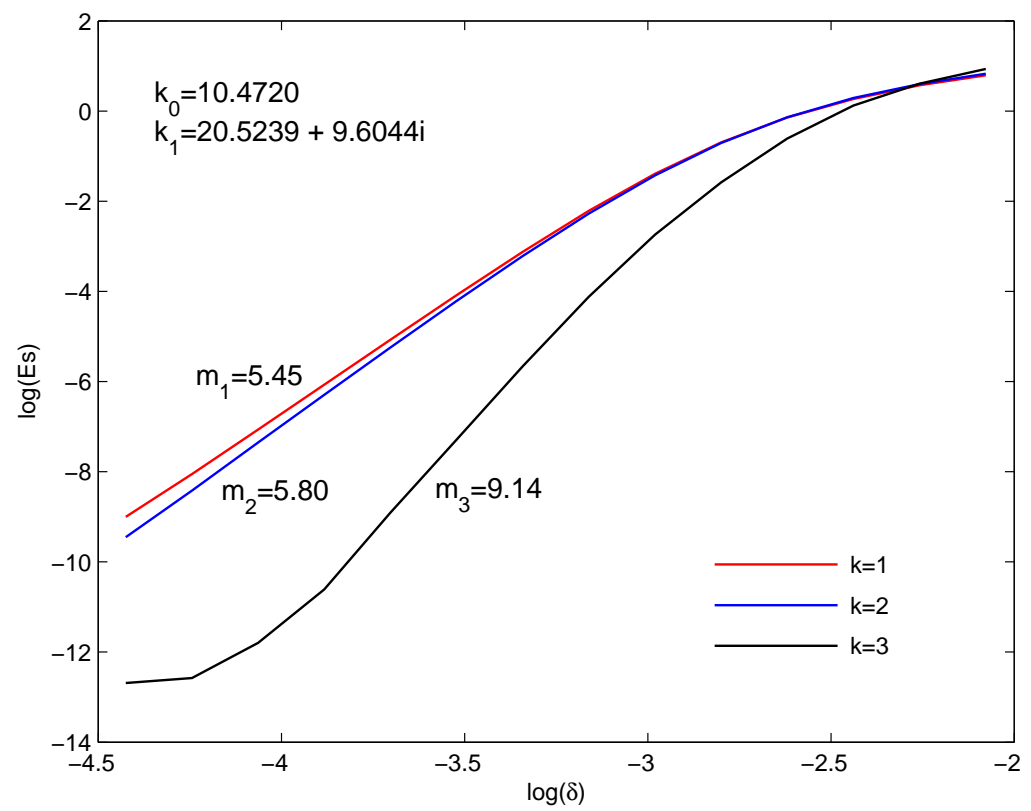

Figure 21: Ex 9a. The graphics of $\log (E s)$ given in (89) for the coating, whose relative dielectric permittivity is $3 \epsilon_{0}$ and conductivity is $\sigma=0.1 S . m^{-1}$, illustrated in Figure 20 in the Dirichlet case 

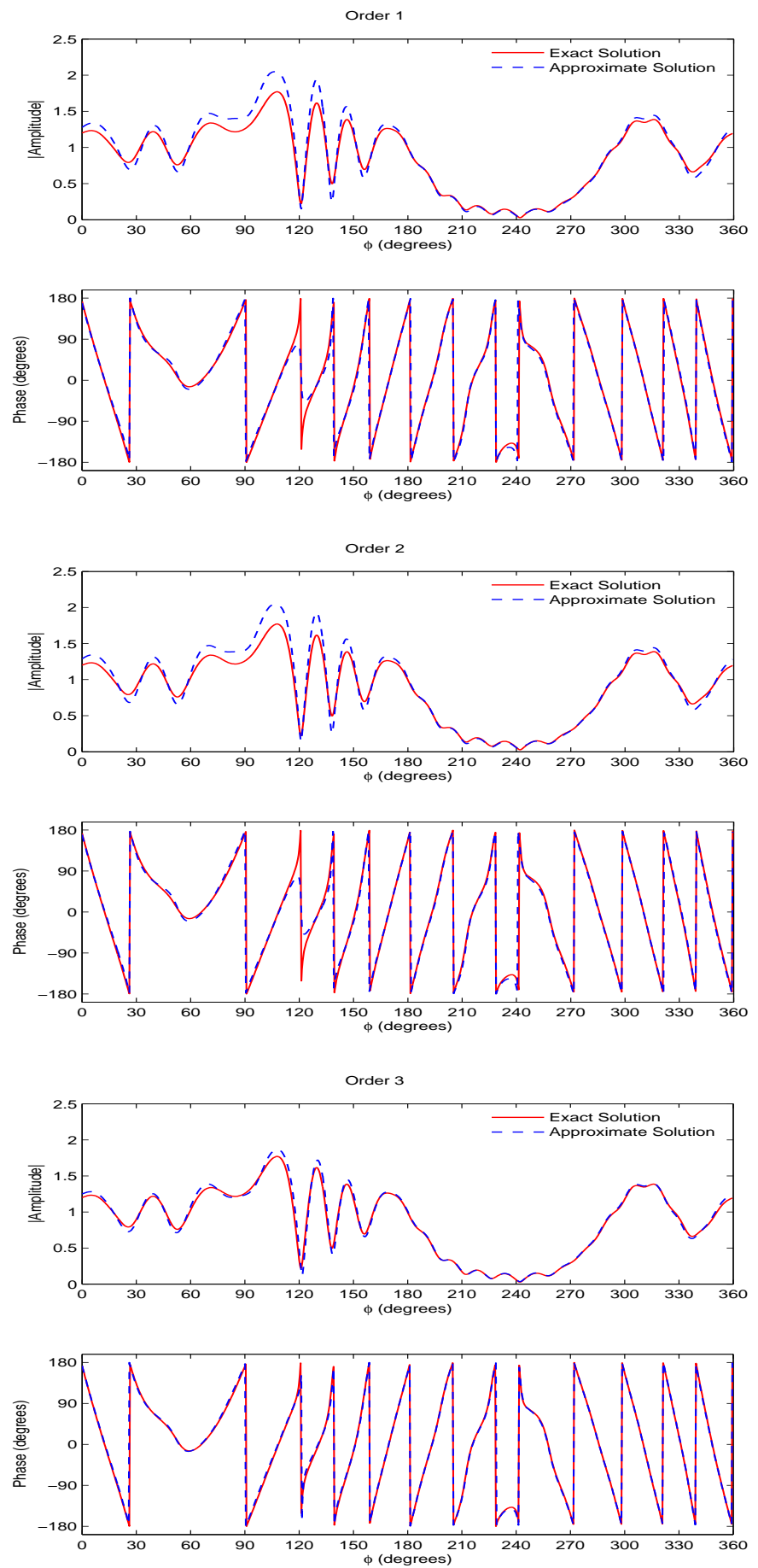

Figure 22: Ex 9. Comparisons of the amplitude and the complex phase of the total field on a circle with radius $\Gamma_{C}=3 \lambda$ obtained by FEM and approximate solutions for the object illustrated in Figure 20 in the Dirichlet case 


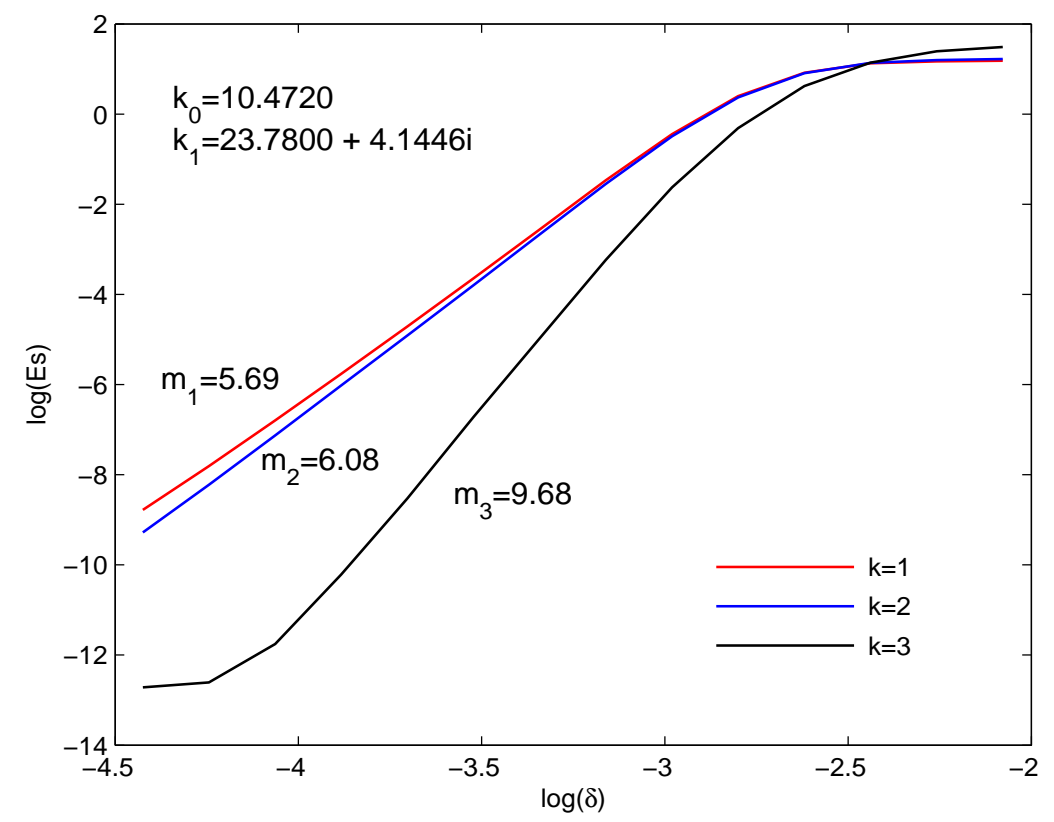

Figure 23: Ex 9b. The graphics of $\log (E s)$ given in (89) for the coating, whose relative dielectric permittivity is $5 \epsilon_{0}$ and conductivity is $\sigma=0.055 . \mathrm{m}^{-1}$, illustrated in Figure 20 in the Dirichlet case 


\subsection{Numerical Results for the Neumann case}

In this section, the numerical solutions of the problem is given to verify the accuracy and effectiveness of the GIBC's for the Neumann case. The exact solution and approximate solutions for order 1,2,3 are compared for various examples.

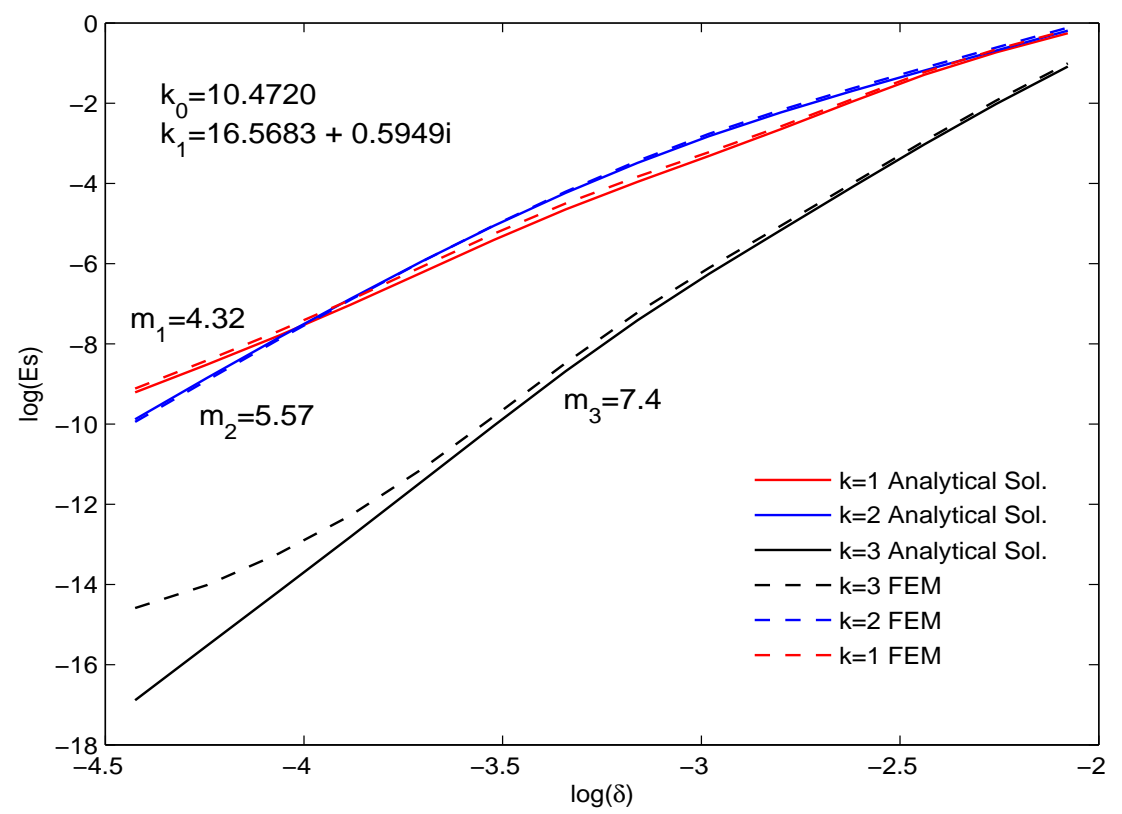

Figure 24: Ex 1. The graphics of $\log (E s)$ given in (89) for the circular coating with radius $5 \lambda / 6$ and constant width in the Neumann case

As a first example, a PEC cylinder coated by a cylinder material of radius $5 \lambda / 6$ with relative dielectric permittivity of $2.5 \epsilon_{0}$ and conductivity of $0.005\left(S . \mathrm{m}^{-1}\right)$ is considered. $f(s)$ is selected as 1 . The object is illuminated at $5 \mathrm{MHz}$ with incidence angle $\pi / 4$. The discretization size is roughly $\lambda / 62$, and the radius of the $\mathrm{ABC}$ is $2.5 \lambda$. The errors of total field Es's are calculated on the circle $\Gamma_{C}$ with radius $5 \lambda / 3$ and for order 1,2 , and 3 the equation of the lines given in (89) are compared in Figure 24] Furthermore, the lines are derived for analytical solutions to verify the accuracy of FEM. The slope of lines for order 1,2 and 3 are approximately $m_{1}=4.32, m_{2}=5.57$ and $m_{3}=7.4$, respectively.

As a second example, an elliptical cross-sectioned dielectric coating with relative dielectric permittivity of $2.5 \epsilon_{0}$ and conductivity of $0.007\left(S . \mathrm{m}^{-1}\right)$ whose boundary given in 90 where $a=7 \lambda / 6$ and $b=2 \lambda / 3$ is taken into account. The width $\delta(s)$ is constant. The object is illuminated at $5 \mathrm{MHz}$ with incidence angle $\pi / 4$. The discretization size is roughly $\lambda / 59$, and the radius of the $\mathrm{ABC}$ is $2.5 \lambda$. The errors of total field Es's are calculated on the circle $\Gamma_{C}$ with radius $5 \lambda / 3$ and for order 1,2 , and 3 equation of lines given in (89) are compared in Figure 25. The slope of lines for order 1,2 and 3 are approximately $m_{1}=4.06$, $m_{2}=5.52$ and $m_{3}=7.36$, respectively. 


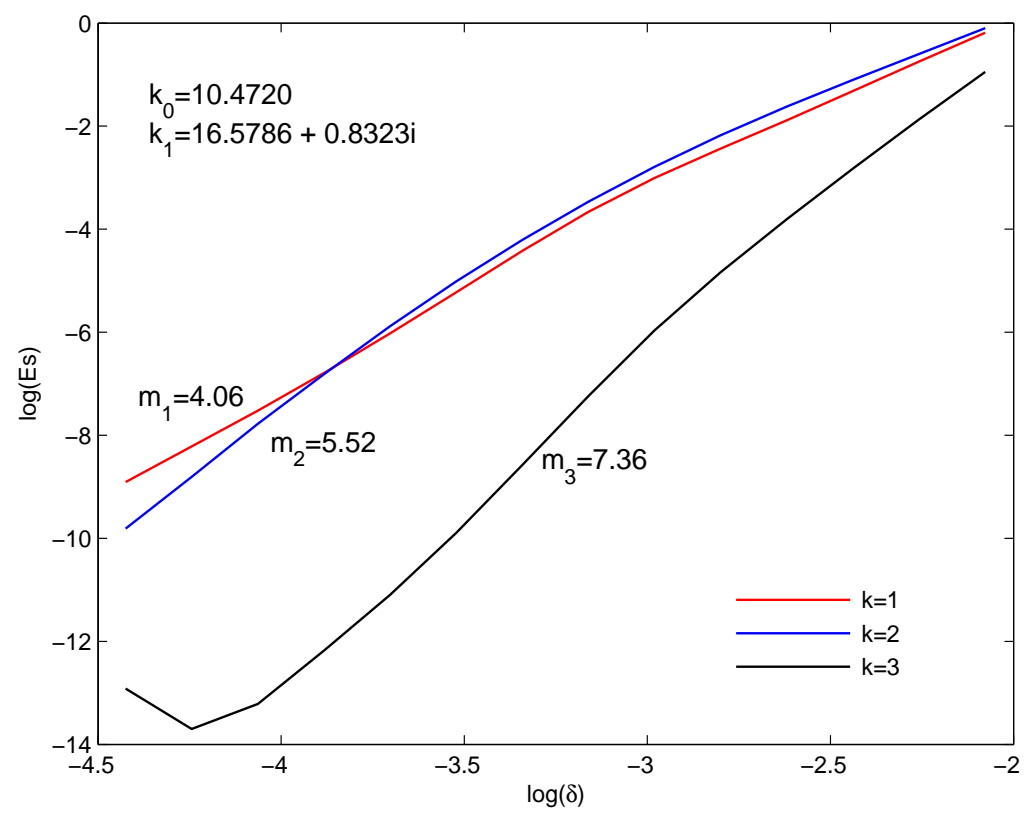

Figure 25: Ex 2. The graphics of $\log (E s)$ given in (89) for the object with constant thickness given by (90) where $a=7 \lambda / 6$ and $b=2 \lambda / 3$ in the Neumann case

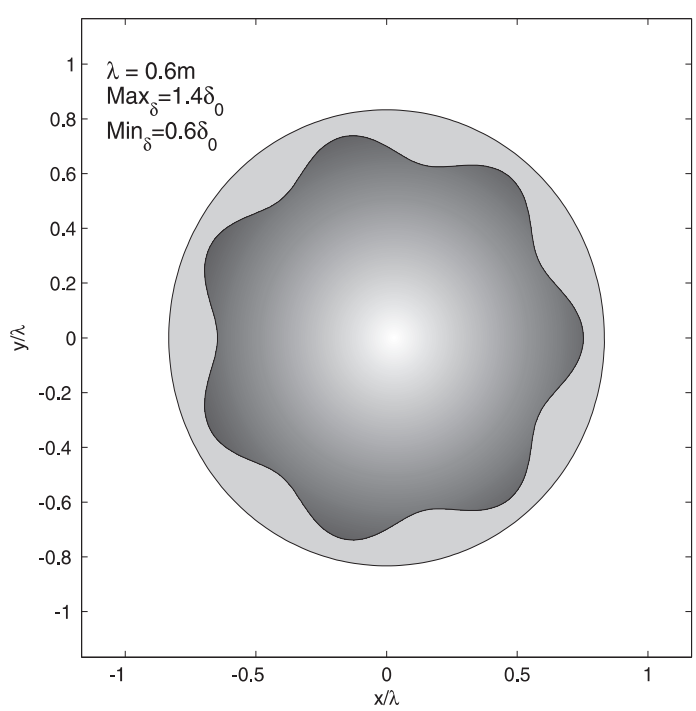

Figure 26: Ex 3. The geometry of the circular coating with radius $5 \lambda / 6$ whose width is given in (103) 
As a third example, a cylindrical cross-sectioned dielectric coating of variable width, with relative dielectric permittivity of $2.0 \epsilon_{0}$ and conductivity of $0.007\left(S . m^{-1}\right)$ is taken into account. The radius of coating is selected as $5 \lambda / 6$ (see Figure 26). The variable of width is given with equation

$$
\delta(t)=\delta_{0}(1-0.4 \sin (7 t))
$$

The object is illuminated at $5 \mathrm{MHz}$ with incidence angle $\pi$. The discretization

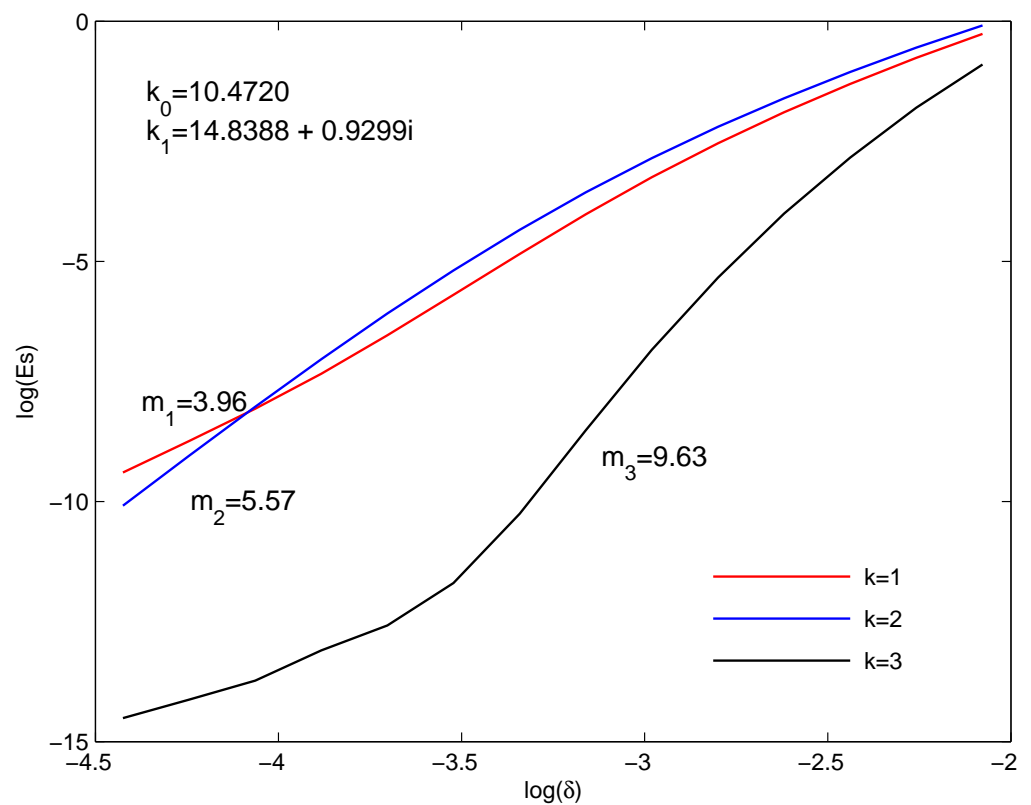

Figure 27: Ex 3. The graphics of $\log (E s)$ given in (89) for the object illustrated in Figure 26] in the Neumann case

size is roughly $\lambda / 62$, and the radius of the $\mathrm{ABC}$ is $2.5 \lambda$. The errors of total field Es's are calculated on the circle $\Gamma_{C}$ with radius $5 \lambda / 3$ and for order 1,2 , and 3 , the equation of the lines are compared in Figure 27. The slope of lines for order 1,2 and 3 are approximately $m_{1}=3.96, m_{2}=5.57$ and $m_{3}=9.63$, respectively.

As a fourth example, an elliptical cross-sectioned dielectric coating of variable width, with relative dielectric permittivity of $3.0 \epsilon_{0}$ and conductivity of $0.008\left(S . \mathrm{m}^{-1}\right)$ is taken into account. The boundary of the coating is given in (90) where $a=1.5 \lambda$ and $b=0.8 \lambda$. The variable of width is given with equation

$$
\delta(t)=\delta_{0}(1-0.4 \sin (7 t))
$$

The object (see Figure 28) is illuminated at $3 \mathrm{MHz}$ with incidence angle $\pi / 3$. The discretization size is roughly $\lambda / 55$, and the radius of the ABC is $2.5 \lambda$. The errors of total field Es's are calculated on the circle $\Gamma_{C}$ with radius $2 \lambda$ and for order 1, 2, and 3, the equation of the lines are compared in Figure 29] The coating is large, curvature of its boundary is variable, and its width is variable, 


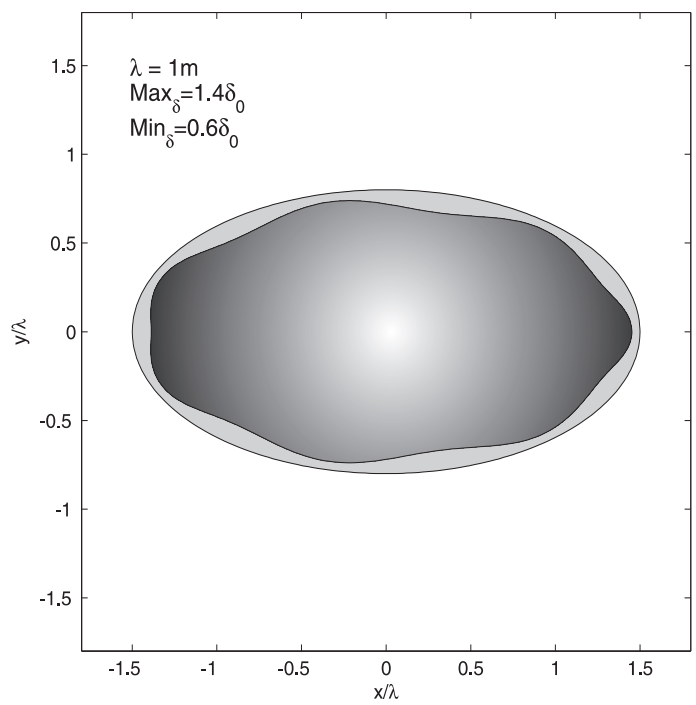

Figure 28: Ex 4. The geometry of the elliptical coating whose boundary is given in (90) where $a=1.5 \lambda, b=0.8 \lambda$ and width is given in (104)

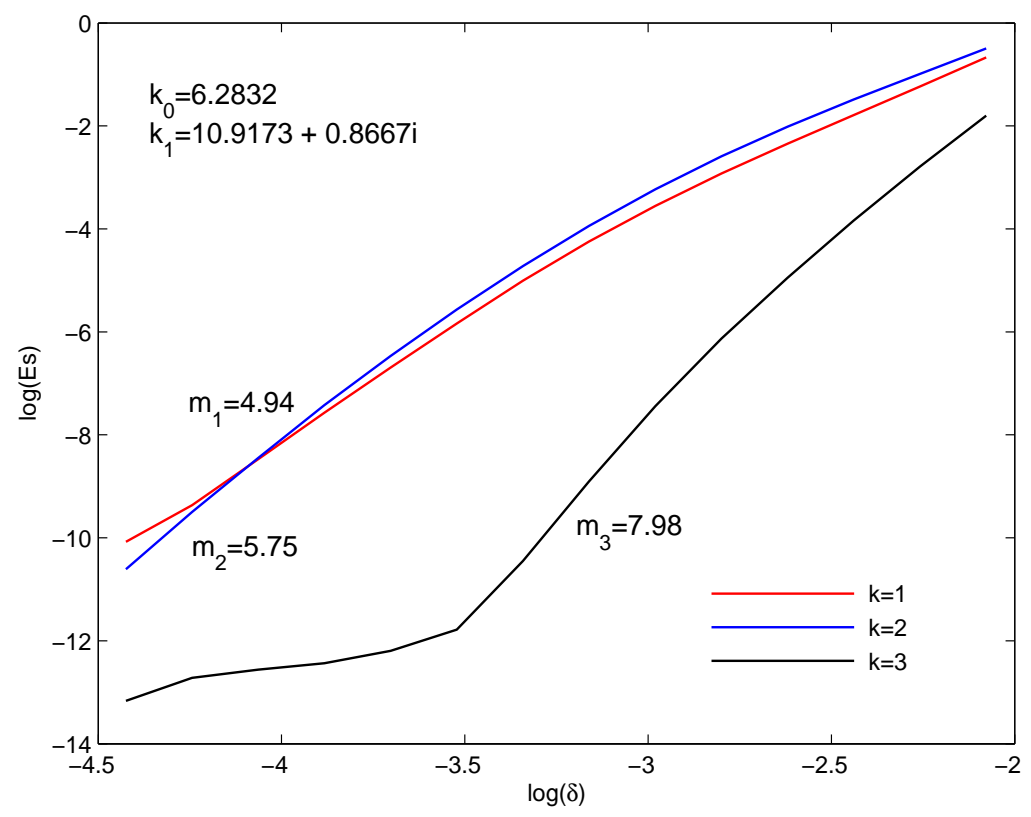

Figure 29: Ex 4. The graphics of $\log (E s)$ given in (89) for the object illustrated in Figure 28 in the Neumann case 
but the expected results are obtained as results of previous examples. The slope of lines for order 1,2 and 3 are approximately $m_{1}=4.94, m_{2}=5.75$ and $m_{3}=7.98$, respectively.

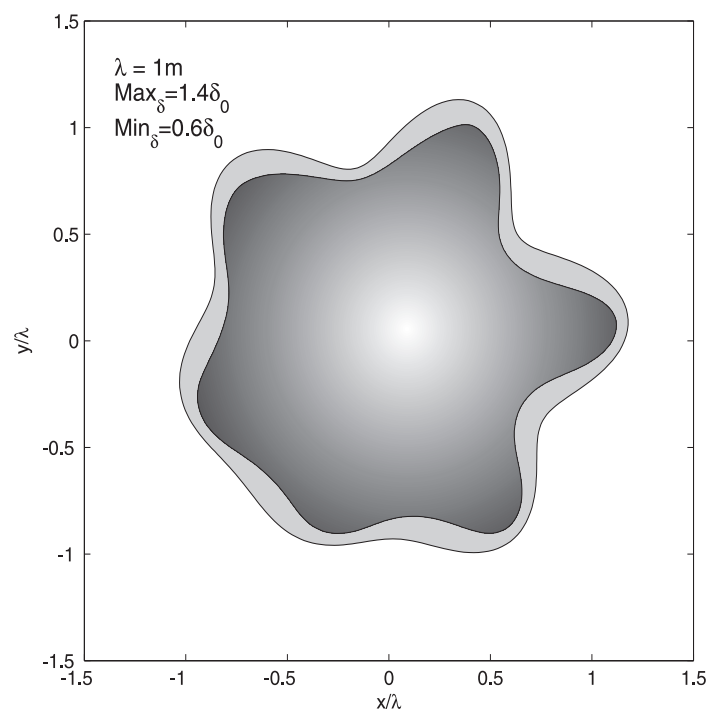

Figure 30: Ex 5. The geometry of the object whose boundary is given in (93) where $A=\lambda, B=0.08 \lambda, C=0.09 \lambda, D=0.07 \lambda$ and width is given in (105)

In the next example, a more complex coating whose boundary is given in (93) where $A=\lambda, B=0.08 \lambda, C=0.09 \lambda$ and $D=0.07 \lambda$ and the width is given by equation

$$
\delta(t)=\delta_{0}(1-0.4 \sin (7 t))
$$

is taken into account(see Figure 30). The coating with relative dielectric permittivity of $2.0 \epsilon_{0}$ and conductivity of $0.006\left(S . \mathrm{m}^{-1}\right)$ is illuminated at $3 \mathrm{MHz}$ with incidence angle $\pi / 4$. The discretization size is roughly $\lambda / 59$, and the radius of the ABC is $2.2 \lambda$. The errors of total field Es's are calculated on the circle $\Gamma_{C}$ with radius $2 \lambda$ and for order 1,2 , and 3 , the equation of the lines are compared in Figure 31] The slope of lines for order 1,2 and 3 are approximately $m_{1}=3.91, m_{2}=5.67$ and $m_{3}=7.83$, respectively.Selecting $\delta_{0}$ as $0.09 \lambda$, the amplitude and the phase of the total field obtained through exact end approximate solutions are compared in Figure 32 The errors are $E_{s}=190.54358^{-3}$, $E_{s}=38.38613 e^{-3}, E_{s}=13.98625 e^{-3}$ and relative errors are $E_{r}=1.67477 e^{-3}$, $E_{r}=0.74428 e^{-3}, E_{r}=0.43892 e^{-3}$, respectively.

As a next example, a kite shaped dielectric coating, with relative dielectric permittivity of $2.5 \epsilon_{0}$ and conductivity of $0.008\left(S . \mathrm{m}^{-1}\right)$, whose boundary is given in (96) where $A=\lambda, B=11 \lambda / 30$ and $C=\lambda / 60$ is taken into account(see Figure 33). The boundary of PEC is given in equation (95) where

$$
\delta(t)=\delta_{0}(1-0.4 \sin (6 t))
$$




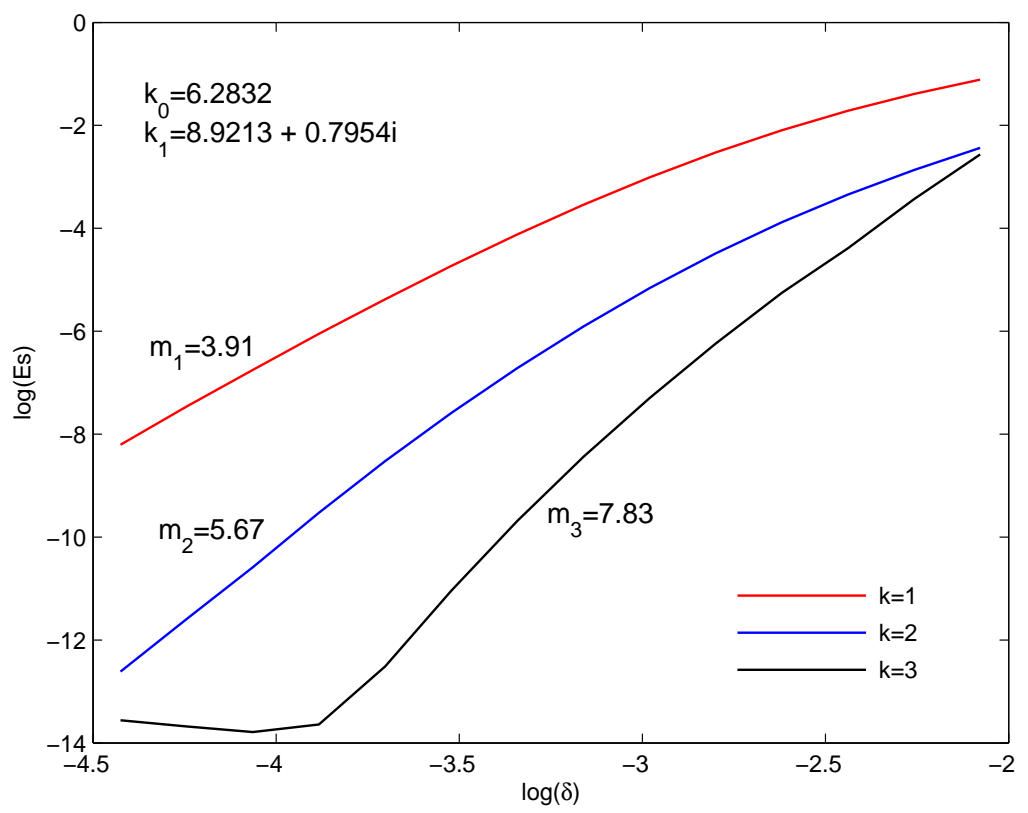

Figure 31: Ex 5. The graphics of $\log (E s)$ given in [89) for the object illustrated in Figure 30] in the Neumann case

The object is illuminated at $5 \mathrm{MHz}$ with incidence angle $\pi / 3$. The discretization size is roughly $\lambda / 56$, and the radius of the $\mathrm{ABC}$ is $8 \lambda / 3$. The errors of total field Es's are calculated on the circle $\Gamma_{C}$ with radius $2 \lambda$ and for order 1,2 , and 3 , the equation of the lines are compared in Figure 34 The slope of lines for order 1,2 and 3 are approximately $m_{1}=4.18, m_{2}=5.3$ and $m_{3}=7.67$, respectively.

As a next example, similar shaped coating whose boundary is given in (96) where $A=0.55 \lambda, B=0.2 \lambda, C=0.01 \lambda$ and width is given in as

$$
\delta(t)=0.08 \lambda(1-0.3 \sin (9 t))
$$

is taken into account(see Figure 18). The coating with relative dielectric permittivity of $4 \epsilon_{0}$ and conductivity of $0.005\left(S . \mathrm{m}^{-1}\right)$ is illuminated at $3 \mathrm{MHz}$ with incidence angle $\pi / 3$. The radius of the $\mathrm{ABC}$ is $1.5 \lambda$. The amplitude and the phase of the total field on the circle $\Gamma_{C}$ with radius $1.2 \lambda$ obtained through exact end approximate solutions are compared in Figure 35] The errors are $E_{s}=160.34480^{-3}, E_{s}=236.09569^{-3}, E_{s}=24.59216 e^{-3}$ and relative errors are $E_{r}=1.53845 e^{-3}, E_{r}=1.84003 e^{-3}, E_{r}=0.60745 e^{-3}$, respectively.

In case of non-regular $\delta(s)$, the same procedure given in previous section is executed. Redefining the expressions over $\Gamma$ as piecewise continuous functions and vanishing the expressions $\delta^{\prime \prime}(s)$, the variational formulations for order 1 , 2 and 3 given in [79, 82, 84 and 86] can be rearranged. One can modify the variational formulation for order 1 (79) as

$$
\int_{\Omega_{-}} \nabla u_{-}^{\delta} \nabla \bar{\nu}-k_{0}^{2} \int_{\Omega_{-}} u_{-}^{\delta} \bar{\nu}
$$



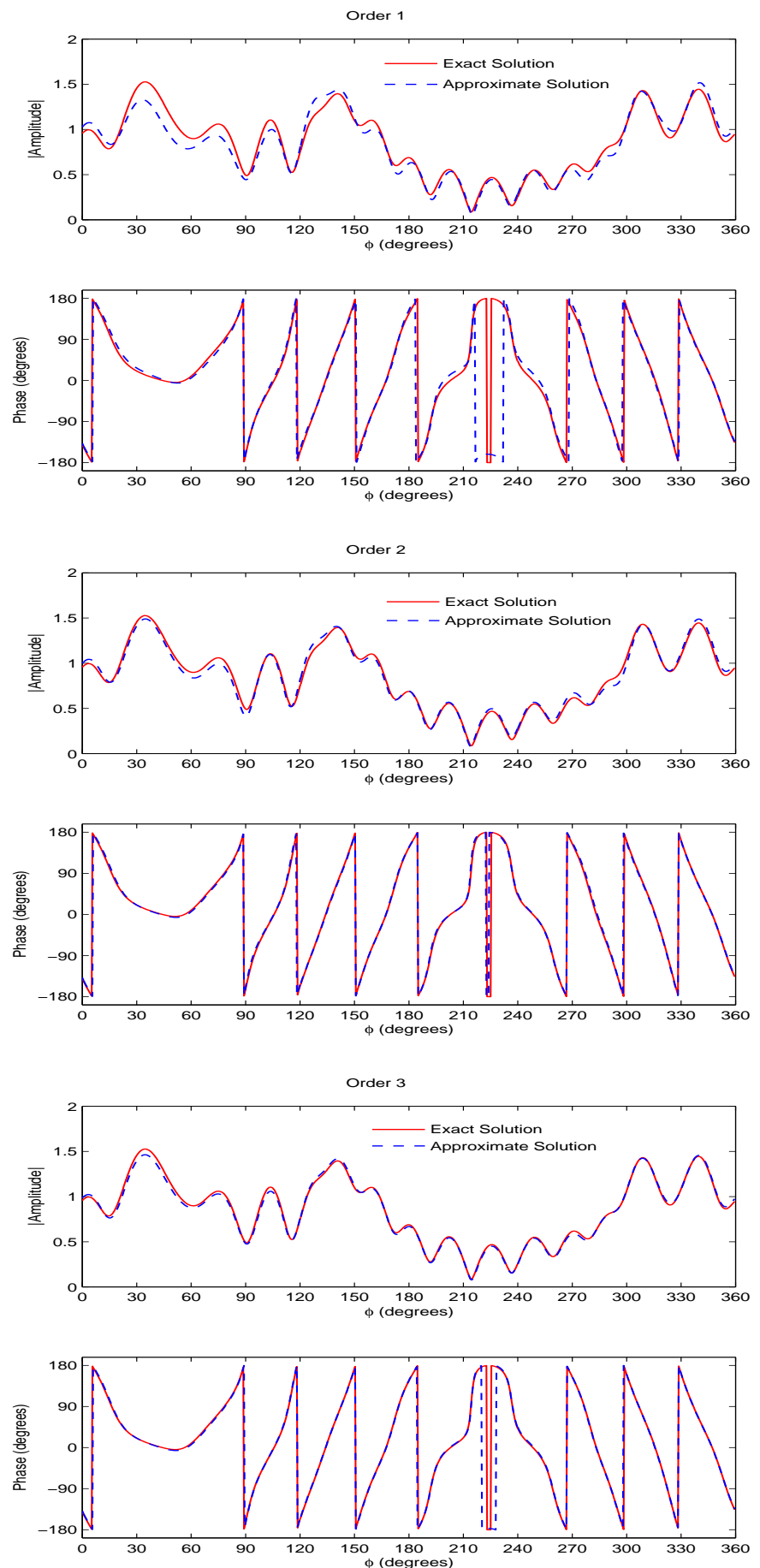

Figure 32: Ex 5. Comparisons of the amplitude and the complex phase of the total field on a circle with radius $\Gamma_{C}=2 \lambda$ obtained by FEM and approximate solutions for the object illustrated in Figure 30] in the Neumann case 


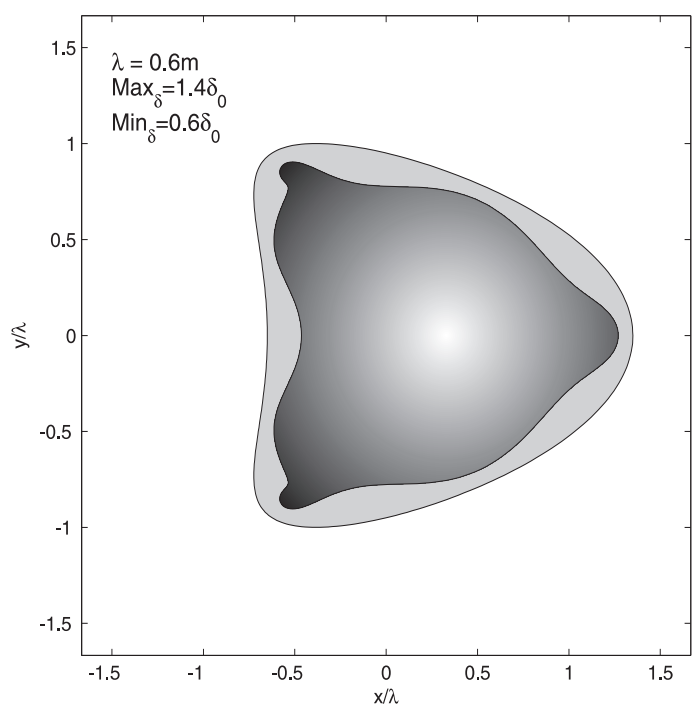

Figure 33: Ex 6. The geometry of the object whose boundary is given in (96) where $A=\lambda, B=11 \lambda / 30, C=\lambda / 60$ and width is given in (106)

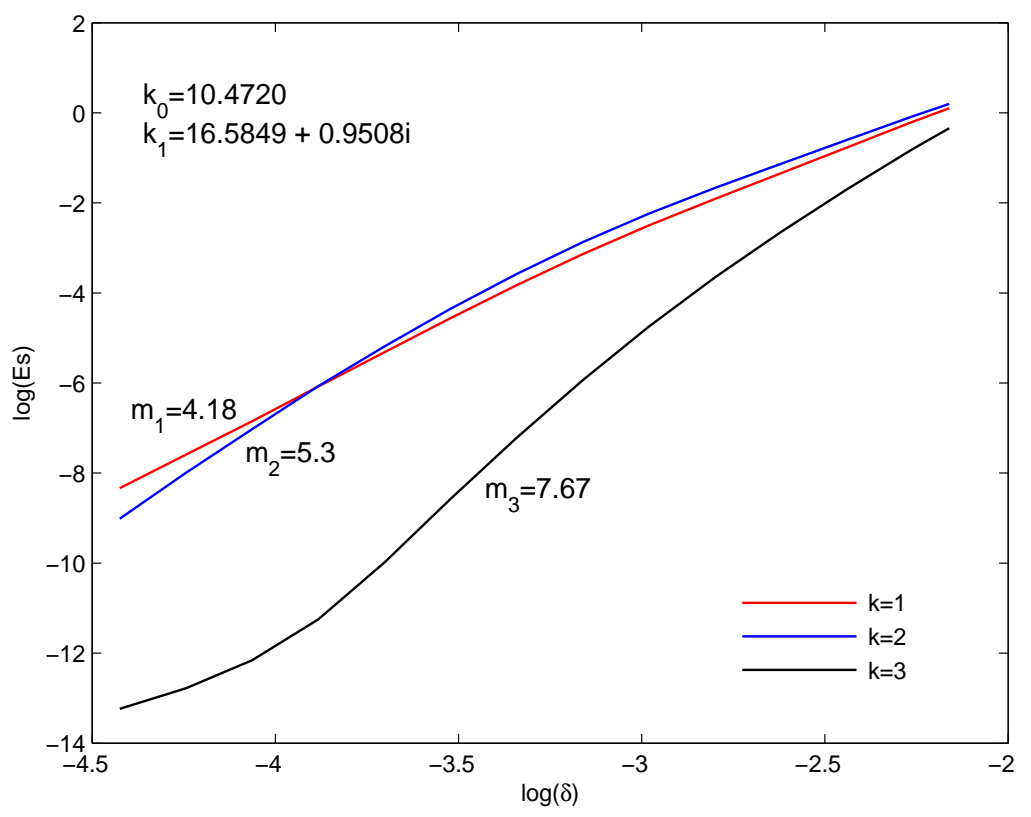

Figure 34: Ex 6. The graphics of $\log (E s)$ given in (89) for the object illustrated in Figure 33 in the Neumann case 

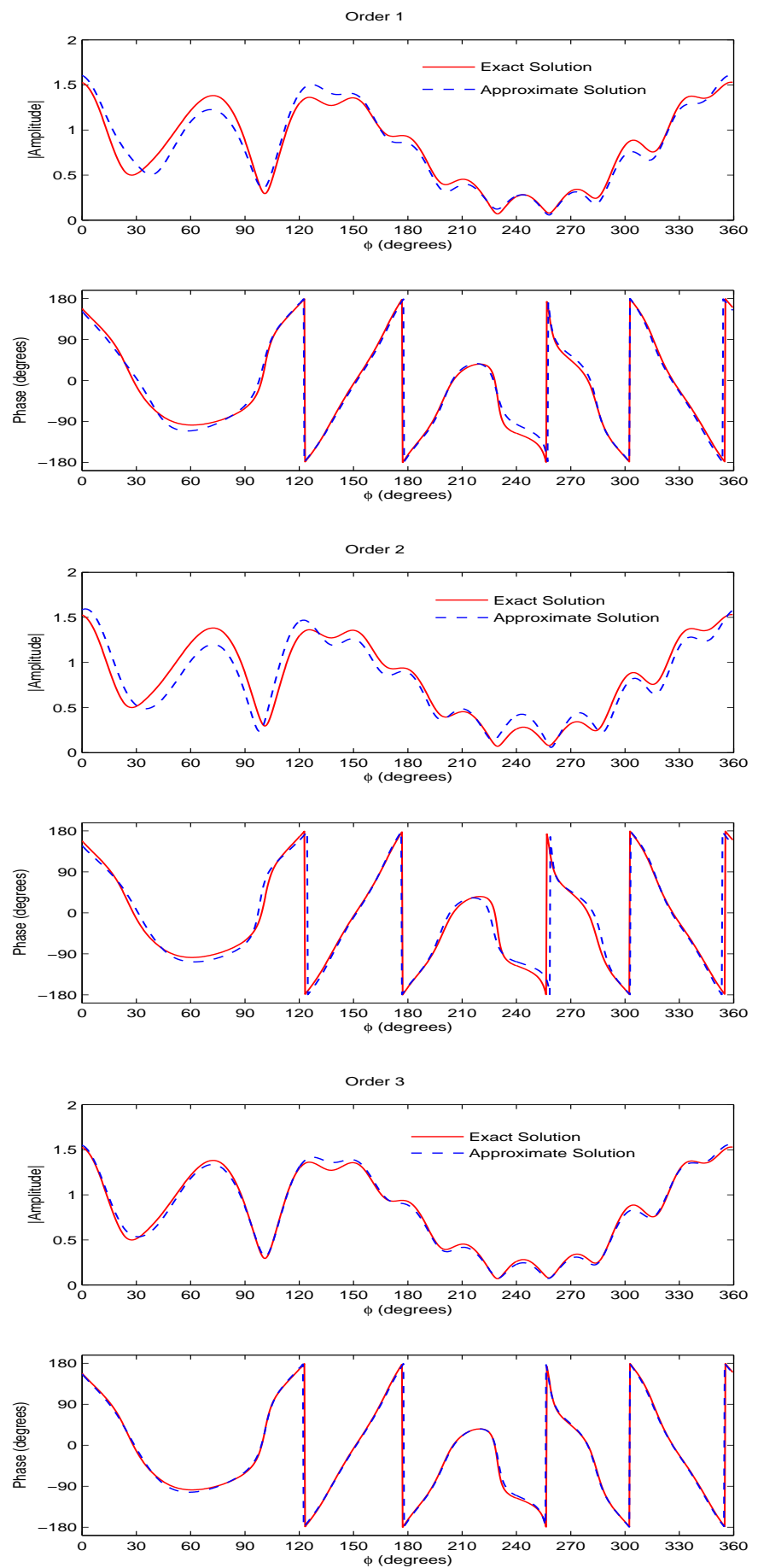

Figure 35: Ex 7. Comparisons of the amplitude and the complex phase of the total field on a circle with radius $\Gamma_{C}=1.2 \lambda$ obtained by FEM and approximate solutions for the object illustrated in Figure [18 in the Neumann case 


$$
\begin{aligned}
-\sum_{n=1}^{N} & \int_{\Gamma_{n}} \delta(s) k_{1}^{2} u_{-}^{\delta} \bar{\nu} d s+\sum_{n=1}^{N} \int_{\Gamma_{n}} \delta(s) \frac{\partial u_{-}^{\delta}}{\partial s} \frac{\partial \bar{\nu}}{\partial s} d s \\
& -\int_{\Gamma_{R}} i k_{0} u_{-}^{\delta} \bar{\nu} d s-\int_{\Gamma_{R}}\left(\frac{\partial u^{i}}{\partial \bar{n}}-i k_{0} u^{i}\right) \bar{\nu} d s=0
\end{aligned}
$$

where $\cup_{1}^{N} \Gamma_{n}=\Gamma$. Similarly, for order 2

$$
\begin{array}{r}
\int_{\Omega_{-}} \nabla u_{-}^{\delta} \nabla \bar{\nu}-k_{0}^{2} \int_{\Omega_{-}} u_{-}^{\delta} \bar{\nu}+\sum_{n=1}^{N} \int_{\Gamma_{n}} \alpha u_{-}^{\delta} \bar{\nu} d s \\
+\sum_{n=1}^{N} \int_{\Gamma_{n}} \gamma \frac{\partial u_{-}^{\delta}}{\partial s} \frac{\partial \bar{\nu}}{\partial s} d s-\int_{\Gamma_{R}} i k_{0} u_{-}^{\delta} \bar{\nu} s-\int_{\Gamma_{R}}\left(\frac{\partial u^{i}}{\partial \bar{n}}-i k_{0} u^{i}\right) \bar{\nu} s=0
\end{array}
$$

is obtained where

$$
\begin{gathered}
\alpha=-k_{1}^{2}\left(\delta(s)+\frac{1}{2} \delta^{2}(s) c(s)\right) \\
\gamma=\delta(s)-\frac{1}{2} \delta^{2}(s) c(s)
\end{gathered}
$$

In case of regular $\delta(s)$, the couple of variational formulation for order 3 has already derived. Analogously, selecting two new variable, the triple of variational formulation is derived. The first variable already has been given in (83) and its variational formulation given in (86) can be modified as

$$
\sum_{n=1}^{N} \int_{\Gamma_{n}} \frac{\partial u_{-}^{\delta}}{\partial s} \frac{\partial \bar{\nu}_{2}}{\partial s} d s+\sum_{n=1}^{N} \int_{\Gamma_{n}} \psi \bar{\nu}_{2} d s=0
$$

The second variable is selected as

$$
\psi_{2}=\frac{1}{2} \delta^{2}(s) \delta^{\prime \prime}(s) \frac{\partial u_{-}^{\delta}}{\partial s}
$$

By partial integration of the $\int_{\Gamma} \frac{1}{2} \delta^{2}(s) \delta^{\prime \prime}(s) \frac{\partial u_{-}^{\delta}}{\partial s} \bar{\nu}_{3} d s$, the expressions $\delta^{\prime \prime}(s)$ can be vanished and new variational formulation related to second variable

$$
\begin{array}{r}
\sum_{n=1}^{N} \int_{\Gamma_{n}} \delta(s) \delta^{\prime}(s)^{2} \frac{\partial u_{-}^{\delta}}{\partial s} \bar{\nu}_{3} d s+\sum_{n=1}^{N} \int_{\Gamma_{n}} \frac{1}{2} \delta^{2}(s) \delta^{\prime}(s) \frac{\partial u_{-}^{\delta}}{\partial s} \frac{\partial \bar{\nu}_{3}}{\partial s} d s \\
+\sum_{n=1}^{N} \int_{\Gamma_{n}} \frac{1}{2} \delta^{2}(s) \delta^{\prime}(s) \psi \bar{\nu}_{3} d s+\sum_{n=1}^{N} \int_{\Gamma_{n}} \psi_{2} \bar{\nu}_{3} d s=0
\end{array}
$$

is obtained where $\bar{\nu}_{3}$ is a test function. Substituting new variables into variational formulation for order 3 given in (84) and vanishing second order of derivatives by partial integration, (84) can be modified as

$$
\begin{array}{r}
\int_{\Omega_{-}} \nabla u_{-}^{\delta} \nabla \bar{\nu}-k_{0}^{2} \int_{\Omega_{-}} u_{-}^{\delta} \bar{\nu}+\sum_{n=1}^{N} \int_{\Gamma_{n}} \alpha u_{-}^{\delta} \bar{\nu} d s \\
+\sum_{n=1}^{N} \int_{\Gamma_{n}} \frac{1}{2} k_{1}^{2} \delta^{2}(s) \delta^{\prime}(s) \frac{\partial u_{-}^{\delta}}{\partial s} \bar{\nu} d s+\sum_{n=1}^{N} \int_{\Gamma_{n}} \frac{1}{2} k_{1}^{2} \delta^{2}(s) \delta^{\prime}(s) u_{-}^{\delta} \frac{\partial \bar{\nu}}{\partial s} d s \\
+\sum_{n=1}^{N} \int_{\Gamma_{n}} \gamma \frac{\partial u_{-}^{\delta}}{\partial s} \frac{\partial \bar{\nu}}{\partial s} d s+\sum_{n=1}^{N} \int_{\Gamma_{n}} \delta^{2}(s) \delta^{\prime}(s) \psi \frac{\partial \bar{\nu}}{\partial s} d s
\end{array}
$$

RR $\mathrm{n}^{\circ} 7145$ 


$$
\begin{array}{r}
+\sum_{n=1}^{N} \int_{\Gamma_{n}} \frac{1}{3} \delta^{3}(s) \frac{\partial \psi}{\partial s} \frac{\partial \bar{\nu}}{\partial s} d s-\sum_{n=1}^{N} \int_{\Gamma_{n}} \frac{\partial \psi_{2}}{\partial s} \bar{\nu} d s \\
-\int_{\Gamma_{R}} i k_{0} u_{-}^{\delta} \bar{\nu} d s-\int_{\Gamma_{R}}\left(\frac{\partial u^{i}}{\partial \bar{n}}-i k_{0} u^{i}\right) \bar{\nu} d s=0
\end{array}
$$

where

$$
\begin{gathered}
\alpha=-k_{1}^{2} \delta(s)\left(1+\frac{1}{2} \delta(s) c(s)+\frac{1}{3} \delta^{2}(s) k_{1}^{2}\right) \\
\gamma=\delta(s)-\frac{1}{2} \delta^{2}(s) c(s)+\frac{1}{3} \delta^{3}(s) c^{2}(s)+\frac{2}{3} \delta^{3}(s) k_{1}^{2}
\end{gathered}
$$

As a last example, dielectric coating of non-regular width is given in previous chapter (see Figure 20) is taken into account. The maximum width and length of the object are $23 \lambda / 6$ and $13 \lambda / 6$, respectively. The object is illuminated at $5 \mathrm{MHz}$ with incidence angle $\pi / 3$. The discretization size is roughly $\lambda / 44$, and the radius of the $\mathrm{ABC}$ is $10 \lambda / 3$. The errors of total field Es's are calculated on the circle $\Gamma_{C}$ with radius $3 \lambda$. When dielectric coating's relative dielectric permittivity and conductivity are selected as respectively $2 \epsilon_{0}$ and $0.005\left(S . m^{-1}\right)$, order 1, 2, and 3 solutions are compared in Figure 36 The slope of lines for order 1,2 and 3 are approximately $m_{1}=4.6, m_{2}=5.58$ and $m_{3}=8.4$, respectively. Selecting $\delta_{0}$ as $\lambda / 12$, the amplitude and the phase of the total field are compared in Figure 37 The errors are $E_{s}=126.73140 e^{-3}, E_{s}=133.55387 e^{-3}$, $E_{s}=7.20718 e^{-3}$ and relative errors are $E_{r}=0.96038 e^{-3}, E_{r}=1.02741 e^{-3}$, $E_{r}=0.23781 e^{-3}$, respectively. Selecting relative dielectric permittivity and

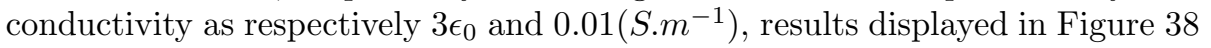
is obtained. The slope of lines are $m_{1}=4.72, m_{2}=5.55$ and $m_{3}=8.3$. 


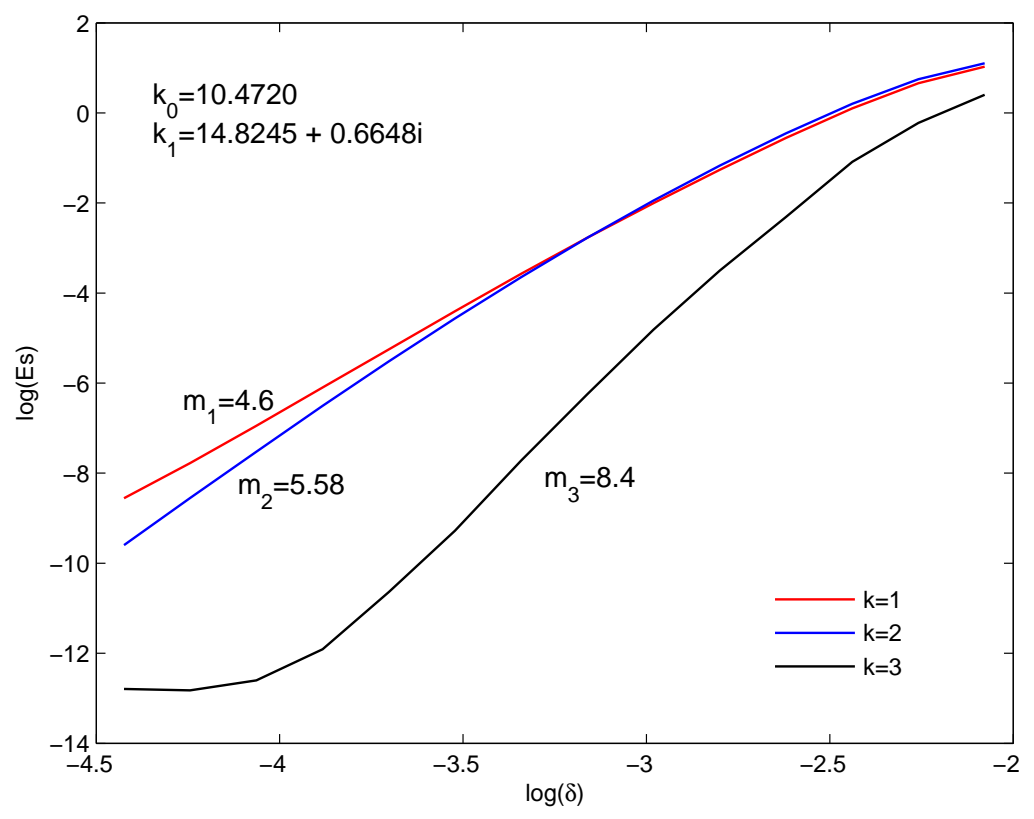

Figure 36: Ex 8a. The graphics of $\log (E s)$ given in 89) for the coating, whose relative dielectric permittivity is $2 \epsilon_{0}$ and conductivity is $\sigma=0.005 . \mathrm{Sm}^{-1}$, illustrated in Figure 20] in the Neumann case 

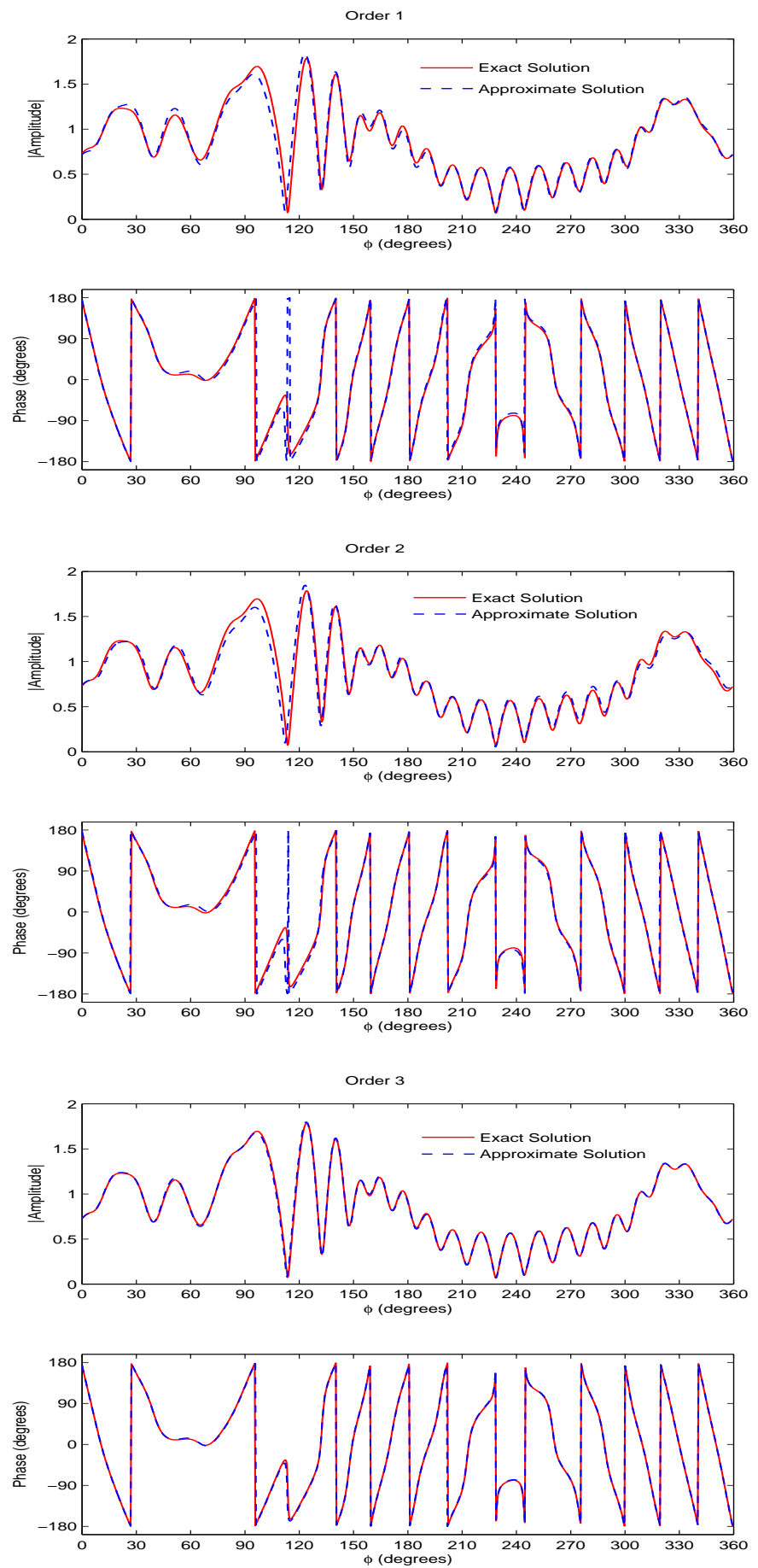

Figure 37: Ex 8. Comparisons of the amplitude and the complex phase of the total field on a circle with radius $\Gamma_{C}=3 \lambda$ obtained by FEM and approximate solutions for the object illustrated in Figure 20] in the Neumann case 


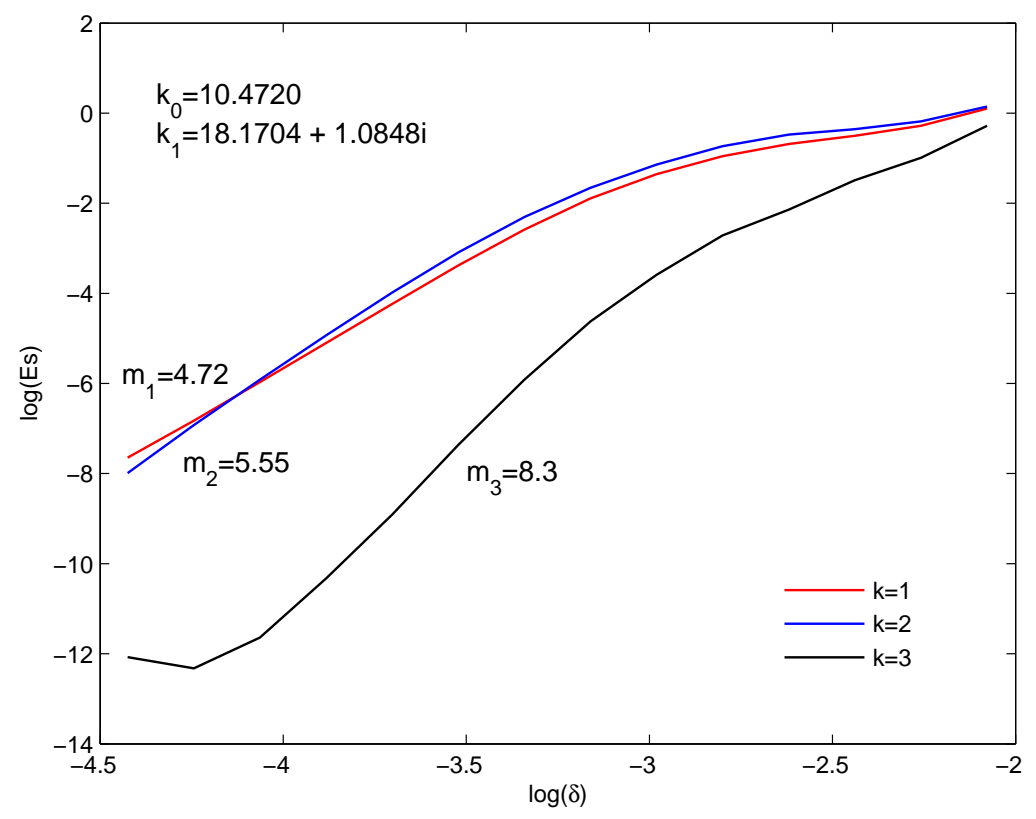

Figure 38: Ex 8b. The graphics of $\log (E s)$ given in (89) for the coating, whose relative dielectric permittivity is $3 \epsilon_{0}$ and conductivity is $\sigma=0.015 . \mathrm{m}^{-1}$, illustrated in Figure 20] in the Neumann case 


\section{Appendix}

\section{Parametrization for curves: used conventions and defini- tions}

Let $\Gamma$ be the boundary of a connected regular domain $\Omega$ and let $x_{\Gamma}=(x(t), y(t))$ be a counterclockwise parametrization of this curve in terms of a parameter $t \in\left[t_{1}, t_{2}\right]$ (this parametrization can be only local). The curvilinear abscissa $\mathrm{s}$

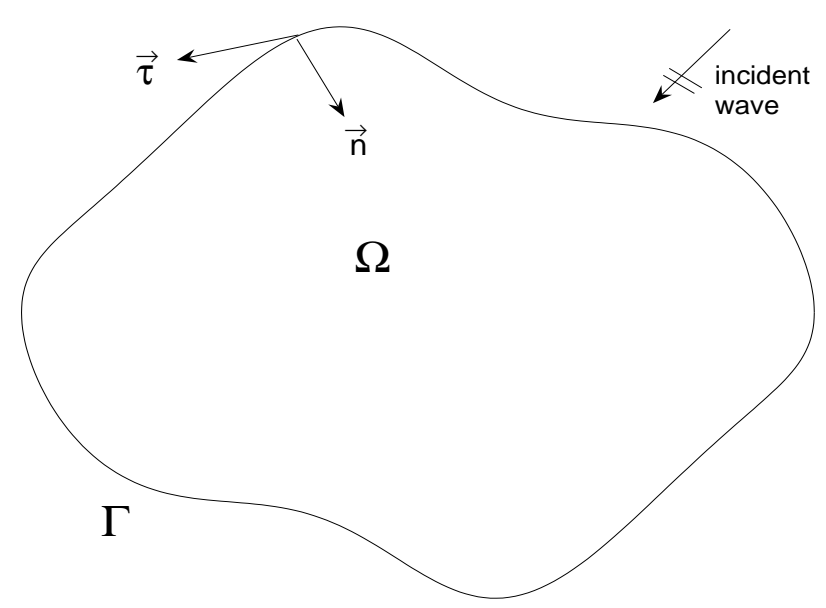

Figure 39: Geometry for the fundamental definitions

is defined as

$$
d s=\sqrt{x^{\prime}(t)^{2}+y^{\prime}(t)^{2}} d t
$$

and the (inward) normal (unitary) is defined by

$$
\vec{n}(t)=\frac{1}{\sqrt{x^{\prime}(t)^{2}+y^{\prime}(t)^{2}}}\left(\begin{array}{r}
-y^{\prime}(t) \\
x^{\prime}(t)
\end{array}\right)
$$

The tangential vector $\vec{\tau}$ is defined by $\vec{\tau}=\frac{d}{d t} x_{\Gamma}$ and is given by

$$
\vec{\tau}(t)=\left(\begin{array}{c}
x^{\prime}(t) \\
y^{\prime}(t)
\end{array}\right)
$$

The curvature $\mathrm{c}(\mathrm{t})$ is defined by

$$
\frac{d \vec{n}}{d t}=c(t) \vec{\tau}
$$

and therefore can be expressed as

$$
c(t)=\frac{x^{\prime \prime}(t) y^{\prime}(t)+y^{\prime \prime}(t) x^{\prime}(t)}{\left(x^{\prime}(t)^{2}+y^{\prime}(t)^{2}\right)^{3 / 2}}
$$


Examples: For a circle object parametric equations are given by

$$
\left\{\begin{array}{l}
x(t)=s \cos (t) \\
y(t)=s \sin (t)
\end{array}\right.
$$

Respectively, the curvature, tangential and normal derivatives are given by

$$
\begin{gathered}
c(t)=-\frac{1}{s} \\
\vec{\tau}=s \vec{e}_{\theta} \\
\vec{n}=-\vec{e}_{s}
\end{gathered}
$$

For an elliptical body parametric equations are given by

$$
\left\{\begin{array}{l}
x(t)=a \cos (t) \\
y(t)=b \sin (t)
\end{array}, a>0, b>0\right.
$$

and the curvature is given by

$$
c(t)=\frac{-a b}{\left(a^{2} \sin ^{2}(t)+b^{2} \cos ^{2}(t)\right)^{3 / 2}}
$$

\section{Acknowledgments}

B. Aslanyürek acknowledge the Scientific and Technological Research Council of Turkey (TUBITAK) for the financial support through Ph.D. scholarships.

\section{References}

[1] A. Bendali and K. Lemrabet. The effect of a thin coating on the scattering of a time-harmonic wave for the Helmholtz equation. SIAM J. Appl. Math., 56(6):1664-1693, 1996.

[2] M. Duruflé, H. Haddar, and P. Joly. Higher order generalized impedance boundary conditions in electromagnetic scattering problems. C.R. Physique, $7(5): 533-542,2006$.

[3] H. Haddar and P. Joly. Stability of thin layer approximation of electromagnetic waves scattering by linear and nonlinear coatings. J. Comput. Appl. Math., 143(2):201-236, 2002.

[4] H. Haddar, P. Joly, and H.-M. Nguyen. Generalized impedance boundary conditions for scattering by strongly absorbing obstacles: the scalar case. Math. Models Methods Appl. Sci., 15(8):1273-1300, 2005.

[5] T.B.A. Senior and J.L. Volakis. Approximate boundary conditions in electromagnetics. 41. IEE Electromagnetic waves series, 1995. 
Centre de recherche INRIA Saclay - Île-de-France

Parc Orsay Université - ZAC des Vignes

4, rue Jacques Monod - 91893 Orsay Cedex (France)

Centre de recherche INRIA Bordeaux - Sud Ouest : Domaine Universitaire - 351, cours de la Libération - 33405 Talence Cedex Centre de recherche INRIA Grenoble - Rhône-Alpes : 655, avenue de l'Europe - 38334 Montbonnot Saint-Ismier

Centre de recherche INRIA Lille - Nord Europe : Parc Scientifique de la Haute Borne - 40, avenue Halley - 59650 Villeneuve d'Ascq Centre de recherche INRIA Nancy - Grand Est : LORIA, Technopôle de Nancy-Brabois - Campus scientifique 615, rue du Jardin Botanique - BP 101 - 54602 Villers-lès-Nancy Cedex

Centre de recherche INRIA Paris - Rocquencourt : Domaine de Voluceau - Rocquencourt - BP 105 - 78153 Le Chesnay Cedex

Centre de recherche INRIA Rennes - Bretagne Atlantique : IRISA, Campus universitaire de Beaulieu - 35042 Rennes Cedex

Centre de recherche INRIA Sophia Antipolis - Méditerranée : 2004, route des Lucioles - BP 93 - 06902 Sophia Antipolis Cedex 